\title{
Dynamic Modeling and Adaptive Robust Synchronous Control of Parallel Robotic Manipulator for Industrial Application
}

\author{
Haiqiang Zhang $\mathbb{D}^{1,2}$ Hairong Fang $\mathbb{D},{ }^{1}$ Qi Zou, ${ }^{2}$ and Dan Zhang ${ }^{2}$ \\ ${ }^{1}$ School of Mechanical, Electronic and Control Engineering, Beijing Jiaotong University, Beijing, China \\ ${ }^{2}$ Lassonde School of Engineering, York University, Toronto, Ontario M3J 1P3, Canada \\ Correspondence should be addressed to Haiqiang Zhang; haiqiang@yorku.ca
}

Received 29 July 2019; Revised 28 October 2019; Accepted 14 November 2019; Published 7 February 2020

Guest Editor: Kiyong Oh

Copyright (C) 2020 Haiqiang Zhang et al. This is an open access article distributed under the Creative Commons Attribution License, which permits unrestricted use, distribution, and reproduction in any medium, provided the original work is properly cited.

\begin{abstract}
Control of parallel manipulators is very hard due to their complex dynamic formulations. If part of the complexity is resulting from uncertainties, an effective manner for coping with these problems is adaptive robust control. In this paper, we proposed three types of adaptive robust synchronous controllers to solve the trajectory tracking problem for a redundantly actuated parallel manipulator. The inverse kinematic of the parallel manipulator was firstly developed, and the dynamic formulation was further derived by mean of the principle of virtual work. Furthermore, linear parameterization regression matrix was determined by virtue of command function "equationsToMatrix" in MATLAB. Secondly, the three adaptive robust synchronous controllers (i.e., sliding mode control, high gain control, and high frequency control) are developed, by incorporating the camera sensor technique into adaptive robust synchronous control architecture. The stability of the proposed controllers was proved by utilizing Lyapunov theory. A sequence of simulation tests were implemented to prove the performance of the controllers presented in this paper. The three proposed controllers can theoretically guarantee the errors including trajectory tracking errors, synchronization errors, and cross-coupling errors asymptotically converge to zero for a given trajectory, and the estimated unknown parameters can also approximately converge to their actual values in the presence of unmodeled dynamics and external uncertainties. Moreover, all the simulation comparative results were presented to illustrate that the adaptive robust synchronous high-frequency controller possess a much superior comprehensive performance than two other controllers.
\end{abstract}

\section{Introduction}

Compared to counterpart serial manipulators, parallel manipulators exhibit much greater advantages such as high stiffness, lower inertia, high loading capability, high precision, high acceleration, and high dexterity, which are desirable properties for application in high-speed machine, high-precision-assisted surgery, and high-speed pick and place of Delta robots, and some other applications [1-4]. However, small workspace and abundant singularities within the workspace limit their wide applications. Under such circumstances, parallel manipulators with redundant actuation are expected to tackle their shortcomings, as redundant actuation can eliminate or decrease singularities, increase the dexterous workspace, optimize the configuration of driving force, and so on. At present, the research studies on redundant actuation mainly focus on the singularities and the workspace $[5,6]$. However, the intelligent control strategies in the existing literature are seldom studied, which just have gotten more attention to make the most use of their advantages, especially for highspeed, high-accuracy machine tools. Some advanced intelligent control strategies, such as adaptive control and robust control, are very essential for more prosperous application in industries [7-11].

Redundant actuation can be achieved with two different methods, one is to add the kinematic chain including nonconstrained subchains or constrained subchains without increasing the degree of freedom, and the other is to change the passive joint into active joint [12]. Liu et al. [13] studied 
exhaustively the force/motion transmissibility of redundantly actuated parallel manipulators, and six simulation examples illustrate that the actuation redundancy can significantly improve the kinematic performance and enlarge the singularity-free workspace. Yao et al. [14] presented a redundant actuated 5UPS-PRPU parallel manipulator by adding an actuator to the middle PRPU passive constraint branch to form a redundant branch; the simulations illustrated that redundant actuation can greatly improve the performance of the parallel manipulator. Boudreau et al. $[15,16]$ presented the dynamic model of a cinematically redundant planar parallel manipulator and optimized to make the actuator torques minimize when the end-effector is performing the trajectory tracking. Wang et al. [17] derived the inverse dynamic model of a spatial 3-DOF parallel manipulator with redundant actuation by adding actuators to passive rotational joints, and the driving force was optimized in terms of the Moore-Penrose inverse matrix. The motion control is very complicated for the coupled dynamics of the parallel manipulator with redundant actuation. The preliminary success of controllers design resulted from planar manipulator and Stewart platform with different adaptive control methods. Ren et al. [18] proposed a novel adaptive synchronized control for a planar parallel manipulator with parametric uncertainty and applied it to improve the tracking accuracy. Shang and Cong [19] developed a nonlinear adaptive controller in the task space for the trajectory tracking of a 2-DOF redundantly actuated parallel manipulator, and the control law including dynamics compensation, adaptive friction compensation and error elimination items was designed simultaneously. In [20], Babaghasabha et al. addressed the design and implementation of adaptive control on a planar cable-driven parallel manipulator with uncertainties in dynamic and kinematic parameters based on simplified hypothesis. Zhang and Wei [21] indicated that adaptive control is generally divided into three categories, model reference control, selftuning control and gain schedule control, and presented a review and discussion on the model reference control of parallel manipulator. Wang et al. [22] proposed an adaptive back-stepping technique for point control of a planar parallel robot, and experimental results showed that the adaptive back-stepping controller outperforms all the other controllers (mentioned in the paper, i.e., back-stepping controller, adaptive controller, adaptive PD controller, and $\mathrm{PD}$ controller) in terms of steady-state errors. Honegger et al. [23] proposed a nonlinear adaptive control algorithm and conducted parameters identification of a 6-PSS parallel manipulator employed as a high-speed milling machine. Zhao et al. [24] developed a fully adaptive feed-forward feedback synchronized tracking control approach for precision tracking control of six-degree-of-freedom Stewart Platform, and the simulation results illustrated that the proposed cross-coupling approach can guarantee both position error and synchronization error converge to zero asymptotically. Hence, synchronous control is expected to be applied to improve trajectory tracking accuracy.

In recent years, robust control method has been widely employed in dealing with nonlinear control with uncertainties and disturbances [25]. Yime et al. [26] proposed a robust adaptive control for the Stewart Gough platform, and the performance of the control law was evaluated by using a sinusoidal path for position and orientation of the upper platform. Zhu et al. [27] presented an adaptive robust posture controller for a parallel manipulator driven by pneumatic muscles with a redundant degree of freedom, which can be utilized to deal with parametric uncertainties and uncertain nonlinearities in the dynamics. Subsequently, a new adaptive robust control architecture for a class of uncertain Euler-Lagrange system was proposed to apply to the wheeled mobile robot, and the experimental results demonstrated that the proposed controller improves control performance in comparison to the adaptive sliding mode control [28]. The control scheme makes full advantage of the adaptive control theory and parameters identification, which played the key role in adaptive strategy; many effective parameter estimation techniques were integrated into adaptive controller design [29]. In addition, the Lyapunov design method emerged as a popular method for estimation in an adaptive algorithm. In this method, the parameter update law was designed in such a way that the time derivative of Lyapunov function was nonpositive [30]. To achieve the proposed tracking trajectory and chattering phenomenon elimination, a robust control strategy was designed for the robotic manipulator based on the sliding mode function and a continuous adaptive control law. And the robustness and stability of the systems had been verified by the Lyapunov theory in [31].

The motivation of this research is to design a stable adaptive robust synchronous control scheme for tracking control of the parallel manipulator with a guaranteed error convergence and without a requirement for prior knowledge of the dynamics of the parallel manipulator. The main contribution of this paper is proposing an alternative adaptive robust synchronous high-frequency control scheme for a 3-DOF parallel manipulator. The synchronization errors and cross-coupling errors are incorporated into adaptive robust control architecture through position and orientation error compensations. The proposed adaptive robust synchronous high-frequency control scheme can obtain the stable tracking performances and synchronization performances in the presence of external uncertainties. A series of simulations were conducted to demonstrate that the proposed control scheme possesses better comprehensive performance than other controllers.

The reminder of this paper is organized as follows. In Section 2, the structure of the redundantly actuated 2RPU2SPR parallel manipulator (in which $R, P, U$, and $S$ stand for rotational, prismatic, universal, and spherical kinematic joints, respectively, and the underline format $(\mathrm{P})$ represents the actuated joint) and the coordinate frames are developed. The kinematic relations of the parallel manipulator are presented in detail in Section 3. In Section 4, the explicit dynamic model is derived in terms of the principle of virtual work and d'Alembert formulation. Next, in Section 5, three adaptive robust synchronous controllers are introduced and deduced in considerable detail. In Section 6, the comparative simulation cases are given to verify the effectiveness of the 
proposed three controllers. Finally, some concluding remarks and future work are presented in Section 7.

\section{System Description}

The parallel manipulator has three degrees of freedom and mainly consists of the fixed platform, the moving platform, and four limbs with two of the same identical kinematic configuration. The parallel manipulator module is a good candidate for engineering application, what's more, it can connect X-Y linear rail in series to achieve five-axis serialparallel hybrid kinematic machine tool, which can complete the complex special-shaded surface free machining (as shown in Figure 1).

In order to conduct experimental verification analysis, we fabricated a scaled-test prototype (as depicted in Figure 2) whose kernel module is a $2 \mathrm{RPU}-2 \mathrm{SPR}$ parallel manipulator with redundant actuation because the number of actuators is greater than that of the degree of freedom. The structural diagram is depicted in Figure 3. The prismatic joints described by the linear joint variables $\mathbf{d}_{i}$ connect the fixed platform to the moving platform by a rotational joint followed by a universal joint or a spherical joint followed by a rotational joint. It is worth noting that the used spherical joint is designed by means of three mutually perpendicular rotational joints in order to enlarge the rotation range.

For modeling purposes, as shown in Figure 3, we assume a fixed coordinate system $B-x y z$ at the centered point $\mathbf{B}$ of the fixed platform and a moving coordinate system $A-u v w$ on the square moving platform at the centered point $\mathbf{A}$, along the $z$-axis and $w$-axis perpendicular to the platform and the $x$-axis and $y$-axis parallel to the $u$-axis and $v$-axis, respectively. Both $A_{1} A_{2} A_{3} A_{4}$ and $B_{1} B_{2} B_{3} B_{4}$ are designed to be squares to yield a symmetric architecture of the parallel manipulator. With these geometric conditions satisfied, the moving platform of parallel manipulator will possess with three degrees of freedom in the Cartesian space by controlling the movement of four actuators, that is, two rotational degrees of freedom around $x$-axis and $v$-axis, and one translational degree of freedom along $z$ axis; in this case, the spatial rotations are coupled along with the change of four nonidentical limbs. The mobility analysis of the parallel manipulator including initial configuration and general configuration has been addressed in detail in our previous work [32] by resorting to the screw theory and modified Grubler-Kutzbach (G-K) criterion. To avoid repetition, herein, the mobility regarding the parallel manipulator is not described in this paper.

\section{Kinematics Analysis}

The moving platform can move along $z$ direction and rotate around the $x$-axis and $v$-axis with the consideration of mobility characteristics. The homogeneous transformation matrix $\mathbf{T}$ of the position coordinate point $\mathbf{p}$ can be expressed as follows:

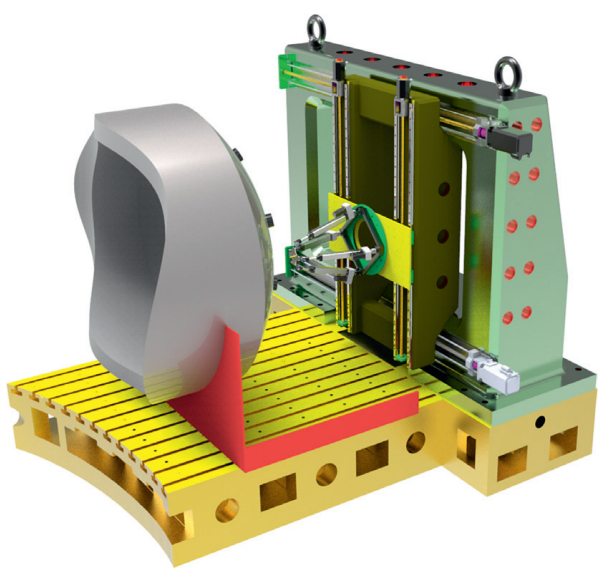

FIgURE 1: A five-axis hybrid kinematic machine tool.

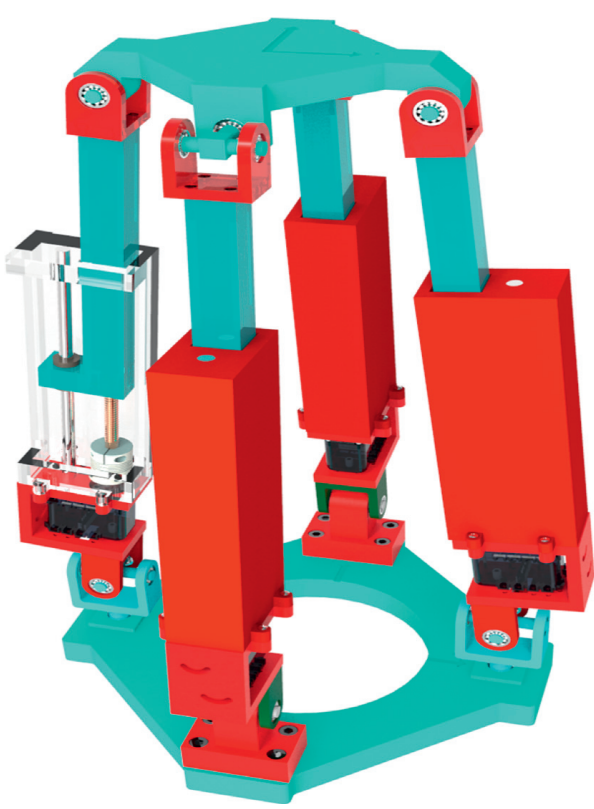

Figure 2: The 3D model of parallel manipulator.

$$
\begin{aligned}
\mathbf{T} & =\left[\begin{array}{ll}
\mathbf{R} & \mathbf{p} \\
0 & 1
\end{array}\right]=\operatorname{Rot}(x, \alpha) \operatorname{Trans}(z, d) \operatorname{Rot}(v, \beta) \\
& =\left[\begin{array}{cccc}
\cos \beta & 0 & \sin \beta & x \\
\sin \alpha \sin \beta & \cos \alpha & -\sin \alpha \cos \beta & y \\
-\sin \beta \cos \alpha & \sin \alpha & \cos \alpha \cos \beta & z \\
0 & 0 & 0 & 1
\end{array}\right],
\end{aligned}
$$

where $\mathbf{p}=[x, y, z]^{\mathrm{T}}$ is the position coordinates of point $\mathrm{A}$ in the fixed coordinate system, $\alpha$ is the rotation angle around the $x$-axis, and $\beta$ is the rotation angle around the $v$-axis.

Additionally, once the rotation matrix $\mathbf{R}$ is obtained, then the rotational Euler angels will be obtained by

$$
\left\{\begin{array}{l}
\alpha=\arctan 2(R(3,2), R(2,2)), \\
\beta=\arctan 2(R(1,3), R(1,1)),
\end{array}\right.
$$




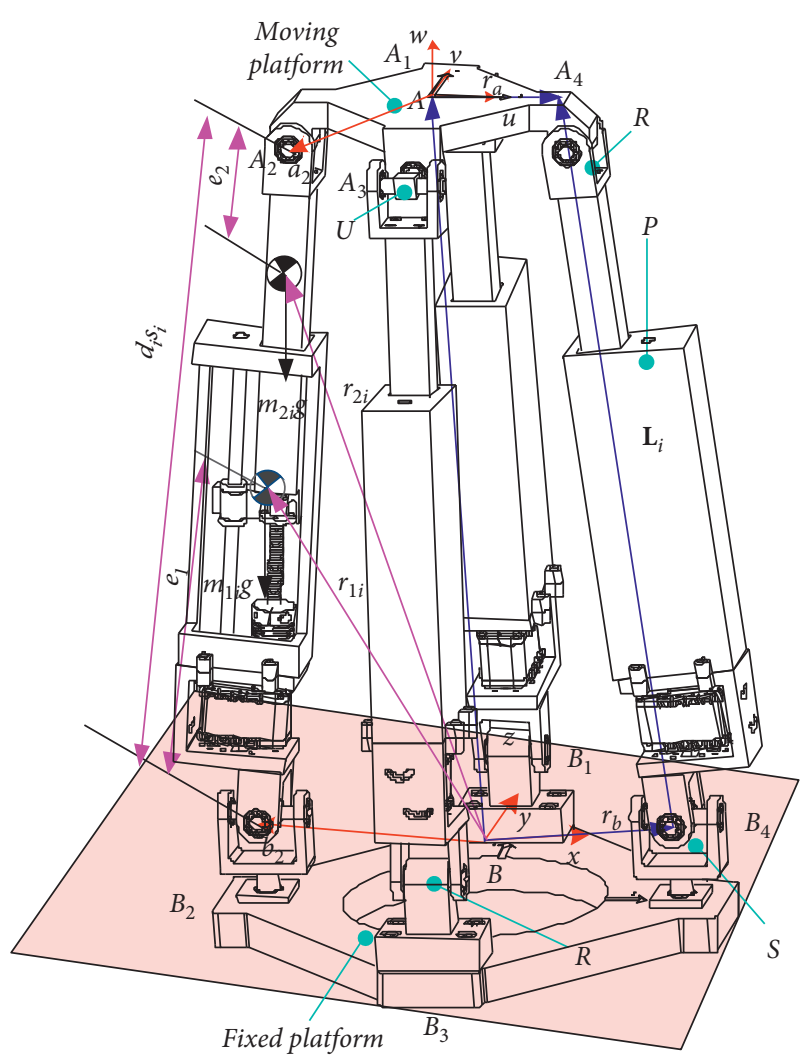

FIGURE 3: Structure diagram of parallel manipulator.

where $R(\cdot, \cdot)$ represents the row and column elements of the rotation matrix $\mathbf{R}$.

Moreover, referring to the aforementioned relations in (1), the coupling relationships can be outlined as

$$
\left\{\begin{array}{l}
x=0 \\
y=-h s \alpha \\
z=h s \alpha
\end{array}\right.
$$

where $h$ is the movement distance along the $z$ direction. So it is very easy to obtain the parasitic motion of the parallel manipulator:

$$
y=-z \tan \alpha
$$

Referring to Figure 3, the closed vector constraint equation of $i$-th limb can be written as

$$
\mathbf{L}_{i}=\mathbf{d}_{i} \cdot \mathbf{s}_{i}=\mathbf{p}+\mathbf{a}_{i}-\mathbf{b}_{i} .
$$

In (5), $\mathbf{b}_{i}$ is the description of the position vector in the fixed coordinate system, and ${ }^{A} \mathbf{a}_{i}$ is the description of the position vector in the moving coordinate system, and $\mathbf{a}_{i}$ is the representation of vector ${ }^{A} \mathbf{a}_{i}$ in the fixed coordinate system, namely, $\mathbf{a}_{i}=\mathbf{R} \cdot{ }^{A} \mathbf{a}_{i}$. To prevent graphics confusion, we only represent $\mathrm{a} 2$ and $\mathrm{b} 2$ in Figure 3 as representative.

The position and orientation of the moving platform can be represented by independent parameters $\mathbf{X}=\left[\begin{array}{lll}z & \alpha & \beta\end{array}\right]^{T}$, the velocity of the $i$-th limb in the inverse kinematics can be derived by differentiating (5) with regard to time and dotmultiplying both sides of (5) by $\mathbf{s}_{i}$, as follows [33]:

$$
\left[\begin{array}{l}
\dot{d}_{1} \\
\dot{d}_{2} \\
\dot{d}_{3} \\
\dot{d}_{4}
\end{array}\right]=\left[\begin{array}{ll}
\mathbf{s}_{1}^{T} & {\left[\mathbf{a}_{1} \times \mathbf{s}_{1}\right]^{T}} \\
\mathbf{s}_{2}^{T} & {\left[\mathbf{a}_{2} \times \mathbf{s}_{2}\right]^{T}} \\
\mathbf{s}_{3}^{T} & {\left[\mathbf{a}_{3} \times \mathbf{s}_{3}\right]^{T}} \\
\mathbf{s}_{4}^{T} & {\left[\mathbf{a}_{4} \times \mathbf{s}_{4}\right]^{T}}
\end{array}\right]\left[\begin{array}{c}
v_{\mathrm{p}} \\
w_{\mathrm{p}}
\end{array}\right],
$$

where $v_{\mathrm{p}}=\left[\begin{array}{ccc}\dot{x} & \dot{y} & \dot{z}\end{array}\right]^{T}$ and $w_{\mathrm{p}}=\left[\begin{array}{lll}w_{x} & w_{y} & w_{z}\end{array}\right]^{T}$ are the linear and angular velocity of the moving platform defined in the fixed coordinate system, respectively.

$$
\left[\begin{array}{l}
w_{x} \\
w_{y} \\
w_{z}
\end{array}\right]=\left[\begin{array}{cc}
1 & 0 \\
0 & \cos \alpha \\
0 & \cos \beta
\end{array}\right]\left[\begin{array}{l}
\dot{\alpha} \\
\dot{\beta}
\end{array}\right] .
$$

It is useful to represent (6) in matrix form as

$$
\dot{\mathbf{q}}=\mathbf{J}_{0} \dot{\mathbf{X}}=\mathbf{J}_{q} \mathbf{J}_{p} \dot{\mathbf{X}},
$$

where $\dot{\mathbf{q}}=\left[\dot{d}_{1}, \dot{d}_{2}, \dot{d}_{3}, \dot{d}_{4}\right]^{T}, \mathbf{J}_{0}$ is the velocity Jacobain matrix of the parallel manipulator, and

$$
\begin{aligned}
\mathbf{J}_{q}= & {\left[\begin{array}{cc}
\mathbf{s}_{1}^{T} & {\left[\mathbf{a}_{1} \times \mathbf{s}_{1}\right]^{T}} \\
\mathbf{s}_{2}^{T} & {\left[\mathbf{a}_{2} \times \mathbf{s}_{2}\right]^{T}} \\
\mathbf{s}_{3}^{T} & {\left[\mathbf{a}_{3} \times \mathbf{s}_{3}\right]^{T}} \\
\mathbf{s}_{4}^{T} & {\left[\mathbf{a}_{4} \times \mathbf{s}_{4}\right]^{T}}
\end{array}\right], } \\
\mathbf{J}_{p}= & {\left[\begin{array}{ccc}
0 & 0 & 0 \\
-\tan \alpha & -z \cdot \sec ^{2} \alpha & 0 \\
\mathbf{J}_{w p}
\end{array}\right]=\left[\begin{array}{ccc}
1 & 0 & 0 \\
0 & 1 & 0 \\
0 & 0 & \cos \alpha \\
0 & 0 & \sin \alpha
\end{array}\right] . }
\end{aligned}
$$

The derivative of independent coordinate parameters can be written in the current case:

$$
\dot{\mathbf{X}}=\left[\begin{array}{lll}
\dot{z} & \dot{\alpha} & \dot{\beta}
\end{array}\right]^{T}
$$

By differentiating both sides of (8), the acceleration between the actuated joints and corresponding end-effector can be expressed as

$$
\ddot{\mathbf{q}}=\dot{\mathbf{J}}_{0} \dot{\mathbf{X}}+\mathbf{J}_{0} \ddot{\mathbf{X}}
$$


where

$$
\dot{\mathbf{J}}_{0}=\left[\begin{array}{ll}
{\left[\mathbf{w}_{1} \times \mathbf{s}_{1}\right]^{T}} & {\left[\left(\mathbf{w}_{p} \times \mathbf{a}_{1}\right) \times \mathbf{s}_{1}+\mathbf{a}_{1} \times\left(\mathbf{w}_{1} \times \mathbf{s}_{1}\right)\right]^{T}} \\
{\left[\mathbf{w}_{2} \times \mathbf{s}_{2}\right]^{T}} & {\left[\left(\mathbf{w}_{p} \times \mathbf{a}_{2}\right) \times \mathbf{s}_{2}+\mathbf{a}_{2} \times\left(\mathbf{w}_{2} \times \mathbf{s}_{2}\right)\right]^{T}} \\
{\left[\mathbf{w}_{3} \times \mathbf{s}_{3}\right]^{T}} & {\left[\left(\mathbf{w}_{p} \times \mathbf{a}_{3}\right) \times \mathbf{s}_{3}+\mathbf{a}_{3} \times\left(\mathbf{w}_{3} \times \mathbf{s}_{3}\right)\right]^{T}} \\
{\left[\mathbf{w}_{4} \times \mathbf{s}_{4}\right]^{T}} & {\left[\left(\mathbf{w}_{p} \times \mathbf{a}_{4}\right) \times \mathbf{s}_{4}+\mathbf{a}_{4} \times\left(\mathbf{w}_{4} \times \mathbf{s}_{4}\right)\right]^{T}}
\end{array}\right] \cdot \mathbf{J}_{p}
$$$$
+\mathbf{J}_{q} \cdot\left[\begin{array}{ccc}
0 & 0 & 0 \\
\left(-1-\tan ^{2} \alpha\right) \cdot \dot{\alpha} & -\left(\dot{z} \cdot \sec ^{2} \alpha+\frac{2 z \cdot \sin \alpha}{\cos ^{3} \alpha} \cdot \dot{\alpha}\right) & 0 \\
0 & 0 & 0 \\
0 & 0 & 0 \\
0 & 0 & -\sin \alpha \cdot \dot{\alpha} \\
0 & 0 & \cos \alpha \cdot \dot{\alpha}
\end{array}\right] \text {, }
$$

where $\mathbf{w}_{i}$ is the angular velocity of the $i$-th limb defined in the fixed coordinate system.

The structural diagram of the $i$-th limb is depicted in Figure 3 as well, each of that consists of an upper sublimb and a lower sublimb. Let $e_{1}$ be the distance between the centroid $B_{i}$ and the center of mass of the lower sublimb, while $e_{2}$ be the distance of the centroid $A_{i}$ and the center of mass of the upper sublimb. The position vectors of the centers of mass of the $i$-th limb are given with respect to the fixed coordinate system:

$$
\begin{aligned}
& \mathbf{r}_{1 i}=\mathbf{b}_{i}+e_{1} \mathbf{s}_{i}, \\
& \mathbf{r}_{2 i}=\mathbf{b}_{i}+\left(\mathbf{d}_{i}-e_{2}\right) \mathbf{s}_{i} .
\end{aligned}
$$

The inverse kinematics (5) should be differentiated with respect to time, and the velocity of kinematic joints $A_{i}$ connected to the moving platform can be obtained in the fixed coordinate system:

$$
\mathbf{v}_{a i}=\mathbf{v}_{p}+\boldsymbol{\omega}_{p} \times \mathbf{a}_{i}=\mathbf{J}_{a i} \cdot \mathbf{J}_{p} \cdot \dot{\mathbf{X}},
$$

where

$$
\mathbf{J}_{a i}=\left[\begin{array}{cccccc}
1 & 0 & 0 & 0 & a_{i z} & -a_{i y} \\
0 & 1 & 0 & -a_{i z} & 0 & a_{i x} \\
0 & 0 & 1 & a_{i y} & -a_{i x} & 0
\end{array}\right] .
$$

The angular velocity of the $i$-th limb

$$
\boldsymbol{\omega}_{i}=\frac{\mathbf{s}_{i} \times \mathbf{v}_{a i}}{\mathbf{d}_{i}}=\mathbf{J}_{w i} \dot{\mathbf{X}},
$$

where $\mathbf{J}_{w i}=\widetilde{\mathbf{s}}_{i} \mathbf{J}_{a i} \mathbf{J}_{p} / \mathbf{d}_{i}$, and $\widetilde{\mathbf{s}}_{i}$ is the antisymmetric operator corresponding to the vector $\mathbf{s}_{i}$.

Taking the derivative of (13) and (14) with respect to time, the velocity of the mass center of the lower sublimb and upper sublimb can be obtained in the fixed coordinate system:

$$
\mathbf{v}_{1 i}=e_{1} \boldsymbol{\omega}_{i} \times \mathbf{s}_{i}=\mathbf{J}_{v 1 i} \dot{\mathbf{X}},
$$

where

$$
\begin{aligned}
\mathbf{J}_{v 1 i} & =-e_{1} \cdot \widetilde{\mathbf{s}}_{i} \mathbf{J}_{w i}, \\
\mathbf{v}_{2 i} & =\left(\mathbf{d}_{i}-e_{2}\right) \boldsymbol{\omega}_{i} \times \mathbf{s}_{i}+\dot{d}_{i} \cdot \mathbf{s}_{i}=\mathbf{J}_{v 2 i} \dot{\mathbf{X}}, \\
\mathbf{J}_{v 2 i} & =\left(e_{2}-\mathbf{d}_{i}\right) \cdot \widetilde{\mathbf{s}}_{i} \mathbf{J}_{w i}+\mathbf{s}_{i} \mathbf{J}_{d i} \mathbf{J}_{p} .
\end{aligned}
$$

Combining (17)-(19), one can obtain

$$
\begin{aligned}
& \mathbf{J}_{v \omega 1 i}=\left[\begin{array}{l}
\mathbf{J}_{v 1 i} \\
\mathbf{J}_{w i}
\end{array}\right], \\
& \mathbf{J}_{v \omega 2 i}=\left[\begin{array}{c}
\mathbf{J}_{v 2 i} \\
\mathbf{J}_{w i}
\end{array}\right],
\end{aligned}
$$

where $\mathbf{J}_{v \omega 1 i}$ and $\mathbf{J}_{v \omega 2 i}$ are the velocity Jacobian matrices of the $i$-th limb.

Turning to accelerations, the acceleration of the point $A_{i}$ can be carried out by differentiating (15) with respect to time:

$$
\dot{v}_{a i}=\dot{\mathbf{v}}_{p}+\dot{\boldsymbol{\omega}}_{p} \times \mathbf{a}_{i}+\boldsymbol{\omega}_{p} \times\left(\boldsymbol{\omega}_{p} \times \mathbf{a}_{i}\right) .
$$

The angular acceleration velocity of the $i$-th limb can be expressed as

$$
\dot{\omega}_{i}=\frac{\mathbf{s}_{i} \times \dot{v}_{a i}-2 \dot{d}_{i} \mathbf{w}_{i}}{\mathbf{d}_{i}}=\mathbf{J}_{w i} \ddot{\mathbf{X}}+\dot{J}_{w i} \dot{\mathbf{X}},
$$

where

$$
\dot{\mathbf{J}}_{w i}=\frac{\widetilde{\mathbf{s}}_{i}\left(\left(\dot{\mathbf{J}}_{v p}-\widetilde{\mathbf{a}}_{i} \dot{\mathbf{j}}_{w p}\right)-\left[\left(\mathbf{J}_{w p} \dot{\mathbf{X}}\right) \times\right] \widetilde{\mathbf{a}}_{i} \mathbf{J}_{w p}\right)-2 \mathbf{J}_{d i} \mathbf{J}_{0} \dot{\mathbf{X}} \mathbf{J}_{w i}}{\mathbf{d}_{i}} .
$$

Finally, the acceleration of the mass centers of the $i$-th limb can be obtained by differentiating once again (18) and (19) with respect to time, respectively,

$$
\dot{v}_{1 i}=e_{1} \dot{\boldsymbol{\omega}}_{i} \times \mathbf{s}_{i}+e_{1} \boldsymbol{\omega}_{i} \times\left(\boldsymbol{\omega}_{i} \times \mathbf{s}_{i}\right)=\mathbf{J}_{v 1 i} \ddot{\mathbf{X}}+\dot{\mathbf{J}}_{v 1 i} \dot{\mathbf{X}},
$$

where

$$
\begin{aligned}
\dot{\mathbf{J}}_{v 1 i} & =-e_{1} \widetilde{\mathbf{s}}_{i} \dot{J}_{w i}-e_{1} \mathbf{s}_{i} \cdot\left(\mathbf{J}_{w i} \dot{\mathbf{X}}\right)^{T} \mathbf{J}_{w i}, \\
\dot{v}_{2 i} & =\ddot{d}_{i} \cdot \mathbf{s}_{i}+2 \dot{d}_{i}\left(\boldsymbol{\omega}_{i} \times \mathbf{s}_{i}\right)+\left(\mathbf{d}_{i}-e_{2}\right) \dot{\boldsymbol{\omega}}_{i} \times \mathbf{s}_{i}+\left(\mathbf{d}_{i}-e_{2}\right) \boldsymbol{\omega}_{i} \times\left(\boldsymbol{\omega}_{i} \times \mathbf{s}_{i}\right) \\
& =\mathbf{J}_{v 2 i} \ddot{\mathbf{X}}+\dot{\mathbf{J}}_{v 2 i} \dot{\mathbf{X}}, \\
\dot{\mathbf{J}}_{v 2 i} & =\mathbf{s}_{i} \cdot \dot{\mathbf{J}}_{0 i}-2 \mathbf{J}_{0 i} \cdot \dot{\mathbf{X}} \cdot \widetilde{s}_{i} \cdot \mathbf{J}_{w i}+\left(e_{2}-\mathbf{d}_{i}\right)\left[\widetilde{\mathbf{s}}_{i} \cdot \dot{\mathbf{J}}_{w i}+\left[\left(\mathbf{J}_{w i} \dot{\mathbf{X}}\right) \times\right] \widetilde{\mathbf{s}} \cdot \mathbf{J}_{w i}\right] .
\end{aligned}
$$

So far, the velocity and acceleration of the moving platform and sublimbs have been solved. In the next section, we will concentrate on the derivation of dynamics of the proposed manipulator.

\section{Dynamics Analysis}

The inverse dynamics problem of the parallel manipulator is to determine the required driving force under the 
requirement generated a prescribed motion trajectory. For the 2RPU-2SPR parallel manipulator, the dynamic model in the Cartesian space can be deduced in the absence of friction and other disturbances by applying the principle of virtual work and d'Alembert formulation stated on the previous work [34], just only the key points are presented, the following dynamic equation can be obtained:

$$
\begin{aligned}
& \mathbf{J}_{0}^{T} \boldsymbol{\tau}_{\mathrm{a}}+\mathbf{J}_{p}\left[\begin{array}{c}
\mathbf{f}_{e}+m_{a} \mathbf{g}-m_{a} \dot{\mathbf{v}}_{p} \\
\mathbf{n}_{e}-{ }^{B} \mathbf{I}_{p} \dot{\boldsymbol{\omega}}_{p}-\boldsymbol{\omega}_{p} \times\left({ }^{B} \mathbf{I}_{p} \boldsymbol{\omega}_{p}\right)
\end{array}\right] \\
& +\sum_{i} \mathbf{J}_{v \omega 1 i}^{T}\left[\begin{array}{c}
m_{1 i} \mathbf{g}-m_{1 i} \dot{\mathbf{v}}_{1 i} \\
-{ }^{B} \mathbf{I}_{1 i} \dot{\boldsymbol{\omega}}_{i}-\boldsymbol{\omega}_{i} \times\left({ }^{B} \mathbf{I}_{1 i} \boldsymbol{\omega}_{i}\right)
\end{array}\right] \\
& +\sum_{i} \mathbf{J}_{v \omega 2 i}^{T}\left[\begin{array}{c}
m_{2 i} \mathbf{g}-m_{2 i} \dot{\mathbf{v}}_{2 i} \\
-{ }^{B} \mathbf{I}_{2 i} \dot{\boldsymbol{\omega}}_{i}-\boldsymbol{\omega}_{i} \times\left({ }^{B} \mathbf{I}_{2 i} \boldsymbol{\omega}_{i}\right)
\end{array}\right]=0 .
\end{aligned}
$$

By simplifying, the dynamics equation of the manipulator can be further deduced as

$$
\begin{aligned}
\mathbf{F}_{X}= & -\left(\mathbf{J}_{p}\left[\begin{array}{c}
\mathbf{f}_{e}+m_{a} \mathbf{g}-m_{a} \dot{\mathbf{v}}_{p} \\
\mathbf{n}_{e}-{ }^{B} \mathbf{I}_{p} \dot{\boldsymbol{\omega}}_{p}-\boldsymbol{\omega}_{p} \times\left({ }^{B} \mathbf{I}_{p} \boldsymbol{\omega}_{p}\right)
\end{array}\right]\right. \\
& +\sum_{i} \mathbf{J}_{v \omega 1 i}{ }^{T}\left[\begin{array}{c}
m_{1 i} \mathbf{g}-m_{1 i} \dot{\mathbf{v}}_{1 i} \\
-{ }^{B} \mathbf{I}_{1 i} \dot{\boldsymbol{\omega}}_{i}-\boldsymbol{\omega}_{i} \times\left({ }^{B} \mathbf{I}_{1 i} \boldsymbol{\omega}_{i}\right)
\end{array}\right] \\
& \left.+\sum_{i} \mathbf{J}_{v \omega 2 i}{ }^{T}\left[\begin{array}{c}
m_{2 i} \mathbf{g}-m_{2 i} \dot{\mathbf{v}}_{2 i} \\
-{ }^{B} \mathbf{I}_{2 i} \dot{\boldsymbol{\omega}}_{i}-\boldsymbol{\omega}_{i} \times\left({ }^{B} \mathbf{I}_{2 i} \boldsymbol{\omega}_{i}\right)
\end{array}\right]\right),
\end{aligned}
$$

where $\mathbf{F}_{X}$ denotes generalized force of the moving platform, ${ }^{B} \mathbf{I}_{1 i}={ }^{B} \mathbf{R}_{i}{ }_{i} \mathbf{I}_{1 i}\left({ }^{B} \mathbf{R}_{i}\right)^{T}$, and ${ }^{B} \mathbf{I}_{2 i}={ }^{B} \mathbf{R}_{i}{ }_{i} \mathbf{I}_{2 i}\left({ }^{B} \mathbf{R}_{i}\right)^{T}$.

In the mentioned above, ${ }^{i} \mathbf{I}_{1 i}$ and ${ }^{i} \mathbf{I}_{2 i}$ stand for the moment of inertia matrix of the lower limb and the upper limb expressed in their own local coordinate system, respectively.

Equation (27) is a dynamics equation of the parallel manipulator, which can be simplified into the general form

$$
\mathbf{F}_{X}=\mathbf{M}(\mathbf{X}) \ddot{\mathbf{X}}+\mathbf{C}(\dot{\mathbf{X}}, \mathbf{X}) \dot{\mathbf{X}}+\mathbf{N}(\mathbf{X}),
$$

where

$$
\begin{aligned}
& \mathbf{M}(\mathbf{X})=\left\{\mathbf{J}_{p}^{T}\left[\begin{array}{c}
m_{a} \mathbf{J}_{v p} \\
{ }^{B} \mathbf{I}_{p} \mathbf{J}_{w p}
\end{array}\right]+\sum_{i}^{4} \mathbf{J}_{v \omega 1 i}^{T}\left[\begin{array}{c}
m_{1 i} \mathbf{J}_{v 1 i} \\
{ }^{B} \mathbf{I}_{1 i} \mathbf{J}_{w i}
\end{array}\right]+\sum_{i}^{4} \mathbf{J}_{v \omega 2 i}^{T}\left[\begin{array}{c}
m_{2 i} \mathbf{J}_{v 2 i} \\
{ }^{B} \mathbf{I}_{2 i} \mathbf{J}_{w i}
\end{array}\right]\right\} \\
& \mathbf{C}(\dot{\mathbf{X}}, \mathbf{X})=\left\{\mathbf{J}_{p}^{T}\left[\begin{array}{c}
m_{a} \dot{\mathbf{J}}_{v p} \\
{ }^{B} \mathbf{I}_{p} \dot{\mathbf{J}}_{w p}+\left[\left(\mathbf{J}_{w p} \dot{\mathbf{X}}\right) \times\right]\left({ }^{B} \mathbf{I}_{p} \mathbf{J}_{w p}\right)
\end{array}\right]+\sum_{i} \mathbf{J}_{v \omega 1 i}{ }^{T}\left[\begin{array}{c}
m_{1 i} \dot{\mathbf{J}}_{v 1 i} \\
{ }^{B} \mathbf{I}_{1 i} \dot{\mathbf{J}}_{v w 1 i}+\left[\left(\mathbf{J}_{v w 1 i} \dot{\mathbf{X}}\right) \times\right]\left({ }^{B} \mathbf{I}_{1 i} \mathbf{J}_{v w 1 i}\right)
\end{array}\right]\right.
\end{aligned}
$$

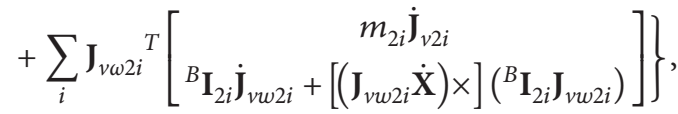

$$
\begin{aligned}
& \mathbf{N}(\mathbf{X})=\mathbf{J}_{p}^{T}\left[\begin{array}{c}
m_{a} \mathbf{g} \\
\mathbf{0}
\end{array}\right]+\sum_{i} \mathbf{J}_{v \omega 1}{ }^{T}\left[\begin{array}{c}
m_{1 i} \mathbf{g} \\
\mathbf{0}
\end{array}\right]+\sum_{i} \mathbf{J}_{v \omega 2}{ }^{T}\left[\begin{array}{c}
m_{2 i} \mathbf{g} \\
\mathbf{0}
\end{array}\right]-\mathbf{J}_{p}^{T}\left[\begin{array}{l}
\mathbf{f}_{e} \\
\mathbf{n}_{e}
\end{array}\right],
\end{aligned}
$$

where $\mathbf{M}(\mathbf{X})$ is the positive definite inertia matrix, $\mathbf{C}(\dot{\mathbf{X}}, \mathbf{X})$ is the nonlinear matrix including the centrifugal and the Coriolis forces term, and $\mathbf{N}(\mathbf{X})$ is the gravitational force and external force term.

Actually, in order to consider the inertial parameter uncertainties and unknown disturbances, the dynamic equation of the parallel robotic manipulator should be written as

$$
\mathbf{F}_{X}=\mathbf{M}(\mathbf{X}) \ddot{\mathbf{X}}+\mathbf{C}(\dot{\mathbf{X}}, \mathbf{X}) \dot{\mathbf{X}}+\mathbf{N}(\mathbf{X})+\boldsymbol{\tau}_{d}^{\prime}
$$

where $\boldsymbol{\tau}_{d}^{\prime}$ is a lumped error vector containing unmodeled dynamics and external disturbances, such as frictions, clearances, wear, noises, and so on.
In addition, the dynamic model has several properties that can be exploited to facilitate dynamic controller design, e.g.,

Property 1. The inertia matrix $\mathbf{M}(\mathbf{X})$ is symmetric and positive definite for any $\mathbf{X} \in R^{n}$.

Property 2. $\dot{\mathbf{M}}(\mathbf{X})-2 \mathbf{C}(\mathbf{X}, \dot{\mathbf{X}})$ is skew symmetric for any $\mathbf{X}$, and the condition should be satisfied

$$
\mathbf{X}^{T}[\dot{\mathbf{M}}(\mathbf{X})-2 \mathbf{C}(\mathbf{X}, \dot{\mathbf{X}})] \mathbf{X}=0 \text {. }
$$

Property 3. The dynamic model can be linearly expressed as

$$
\mathbf{M}(\mathbf{X}) \ddot{\mathbf{X}}+\mathbf{C}(\dot{\mathbf{X}}, \mathbf{X}) \dot{\mathbf{X}}+\mathbf{N}(\mathbf{X})=\mathbf{Y}(\ddot{\mathbf{X}}, \dot{\mathbf{X}}, \mathbf{X}) \Theta
$$


where $\mathrm{Y}$ is a $3 \times 36$ known regressor matrix and $\Theta$ is a $36 \times 1$ unknown parameter vector formed by four parameters for the moving platform and four actuator limbs (upper and lower limbs) of the parallel manipulator. In general, there are four independent perturbation parameters, namely, the mass $m_{i}$ of body and three independent inertia tensors $\mathbf{I}_{x x, \mathrm{i}}, \mathbf{I}_{y y, \mathrm{i}}, \mathbf{I}_{z z, \mathrm{I}}$, i.e.,

$$
\Theta_{i}=\left[\begin{array}{llll}
m_{i} & \mathbf{I}_{x x, i} & \mathbf{I}_{y y, i} & \mathbf{I}_{z z, i}
\end{array}\right]^{T} .
$$

Actually, the regression matrix is very complex due to the coupling relationship [35, 36]; however, it is worth noting that the expressions can be achieved symbolically with the command function "equationsToMatrix" in MATLAB.

Although the dynamics formulation is very complex, the abovementioned three properties are very important to the controller design. Some detail proofs have been addressed in the literature [37].

\section{Controller Design}

With the consideration of the fact that the dynamic formulation of the redundantly actuated parallel manipulator meets the same properties as those of serial robots and nonredundant parallel manipulators, we can apply the control scheme utilized for serial robots and nonredundant parallel manipulators to parallel manipulators with redundant actuation $[38,39]$. In order to achieve a good tracking performance of parallel manipulator in the Cartesian space, an effective advanced control strategy from nonlinear control theory is required. In this paper, we proposed three adaptive robust synchronous controllers to improve the tracking performance of the redundantly actuated parallel manipulator.

The position and orientation tracking error of the task space can be defined as

$$
e_{i}(t)=X_{i}^{d}(t)-X_{i}(t), \quad i=1,2,3,
$$

where $X_{i}^{d}(t)$ and $X_{i}(t)$ denote the desired position and orientation and actual position and orientation, respectively. Besides $e_{i}(t) \longrightarrow 0$, it is aimed to regulate the motion relationships among the mobility during the tracking process.

In order to further improve the tracking accuracy, the motion of moving platform needs to be coordinated to achieve synchronization. Therefore, it is essential to introduce the degree-of-freedom synchronization errors.

To guarantee the tracking error, then the synchronization error [40] can be defined by utilizing the differential error between the corresponding tracking error and the mean value of all the tracking errors:

$$
\varepsilon_{i}=e_{i}-\frac{e_{1}+e_{2}+e_{3}}{N}, \quad N=3 .
$$

Expanding equation (35), one can obtain

$$
\left\{\begin{array}{l}
\varepsilon_{1}=\frac{N-1}{N} e_{1}-\frac{1}{N} e_{2}-\frac{1}{N} e_{3}, \\
\varepsilon_{2}=-\frac{1}{N} e_{1}+\frac{N-1}{N} e_{2}-\frac{1}{N} e_{3}, \\
\varepsilon_{3}=-\frac{1}{N} e_{1}-\frac{1}{N} e_{2}+\frac{N-1}{N} e_{3} .
\end{array}\right.
$$

Obviously, if $e_{i}(t)$ is given, then the synchronization error $\varepsilon_{i}$ can be achieved. Furthermore, we can write equation (36) in the matrix form:

$$
\boldsymbol{\varepsilon}=\mathbf{H}(t) \mathbf{e},
$$

where $\mathbf{e}=\left[\begin{array}{lll}e_{1} & e_{2} & e_{3}\end{array}\right]^{T}$, and $\mathbf{H}(t)$ is the relationship matrix between trajectory tracking errors and synchronization errors.

$$
\mathbf{H}(t)=\frac{1}{N}\left[\begin{array}{ccc}
N-1 & -1 & -1 \\
-1 & N-1 & -1 \\
-1 & -1 & N-1
\end{array}\right]
$$

The cross-coupling error is a compensation term which can reduce tracking error and improve the accuracy of tracking effectively by considering both the restriction and coordination between tracking error and synchronization error. It can be further defined as

$$
\mathbf{e}^{*}=\mathbf{e}+\mathbf{P} \boldsymbol{\varepsilon}+\mathbf{Q} \int_{0}^{t} \boldsymbol{\varepsilon} \mathrm{d} t,
$$

where $\mathbf{P}$ and $\mathbf{Q}$ are the positive definite matrices.

From equation (39), the velocity of cross-coupling error can be obtained by derivative

$$
\dot{\mathbf{e}}^{*}=\dot{\mathbf{e}}+\mathbf{P} \dot{\boldsymbol{\varepsilon}}+\mathbf{Q \varepsilon} .
$$

The reference velocity $\dot{\mathbf{X}}_{\mathrm{r}}$ and acceleration $\ddot{\mathbf{X}}_{\mathrm{r}}$ of the upper platform can be defined as

$$
\left\{\begin{array}{l}
\dot{\mathbf{X}}_{\mathrm{r}}=\dot{\mathbf{X}}_{d}+\mathbf{P} \dot{\boldsymbol{\varepsilon}}+\mathbf{Q} \boldsymbol{\varepsilon}+\Lambda \mathbf{e}^{*}, \\
\ddot{\mathbf{X}}_{\mathrm{r}}=\ddot{\mathbf{X}}_{d}+\mathbf{P} \ddot{\boldsymbol{\varepsilon}}+\mathbf{Q} \dot{\varepsilon}+\Lambda \dot{\mathbf{e}}^{*} .
\end{array}\right.
$$

Obviously, the reference velocity $\dot{\mathbf{X}}_{\mathrm{r}}$ is indeed very important for real-time modification to achieve the desired trajectory and guarantee the convergence of the tracking error.

Furthermore, the reference velocity error including aforementioned hybrid error (i.e., the switched function) can be defined as

$$
\mathbf{S}=\dot{\mathbf{X}}_{\mathrm{r}}-\dot{\mathbf{X}}=\dot{\mathbf{e}}+\mathbf{P} \dot{\boldsymbol{\varepsilon}}+\mathbf{Q} \boldsymbol{\varepsilon}+\Lambda \mathbf{e}^{*} .
$$

The switched function, similar to a sliding surface, can be further simplified considering the relationship (39):

$$
\mathbf{S}=\dot{\mathbf{e}}^{*}-\mathbf{P} \dot{\boldsymbol{\varepsilon}}-\mathbf{Q} \boldsymbol{\varepsilon}+\mathbf{P} \dot{\boldsymbol{\varepsilon}}+\mathbf{Q} \varepsilon+\Lambda \mathbf{e}^{*}=\dot{\mathbf{e}}^{*}+\Lambda \mathbf{e}^{*},
$$

and its differentiation will be

$$
\dot{\mathbf{S}}=\ddot{\mathbf{e}}^{*}+\Lambda \dot{\mathbf{e}}^{*} \text {. }
$$


The robust synchronization control law based on the dynamic feed-forward can be expressed by the following equation:

$$
\mathbf{u}=\widehat{\mathbf{M}} \ddot{\mathbf{X}}_{\mathrm{r}}+\widehat{\mathbf{C}} \dot{\mathbf{X}}_{\mathrm{r}}+\widehat{\mathbf{G}}+\mathbf{K S}+\mathbf{u}_{a u x}
$$

In fact, the control law in abovementioned equation contains three terms, namely, the first term is the dynamic compensation term:

$$
\mathbf{u}_{1}=\widehat{\mathbf{M}} \ddot{\mathbf{X}}_{\mathrm{r}}+\widehat{\mathbf{C}} \dot{\mathbf{X}}_{\mathrm{r}}+\widehat{\mathbf{G}},
$$

where $\widehat{\mathbf{M}}, \widehat{\mathbf{C}}$, and $\widehat{\mathbf{G}}$ are the estimated matrices obtained from the matrices $\mathbf{M}, \mathbf{C}$, and $\mathbf{G}$, respectively.

The second term is the hybrid error compensation term:

$$
\mathbf{u}_{2}=\mathbf{K S},
$$

and it is important to note that the second term can be expanded as

$$
\begin{aligned}
\mathbf{u}_{2} & =\mathbf{K S}=\mathbf{K}\left(\dot{\mathbf{e}}+\mathbf{P} \dot{\boldsymbol{\varepsilon}}+\mathbf{Q} \boldsymbol{\varepsilon}+\Lambda\left(\mathbf{e}+\mathbf{P} \boldsymbol{\varepsilon}+\mathbf{Q} \int_{0}^{t} \boldsymbol{\varepsilon} \mathrm{d} t\right)\right) \\
& =\mathbf{K} \dot{\mathbf{e}}+\mathbf{K} \Lambda \mathbf{e}+\mathbf{K P} \dot{\boldsymbol{\varepsilon}}+(\mathbf{K Q}+\mathbf{K} \Lambda \mathbf{P}) \boldsymbol{\varepsilon}+\mathbf{K} \Lambda \mathbf{Q} \int_{0}^{t} \boldsymbol{\varepsilon} \mathrm{d} t
\end{aligned}
$$

where $\mathbf{u}_{2}$ contains PD control and PID control, and $\mathbf{u}_{2}$ is of crucial importance to improve tracking accuracy performance. In other words, PD control can eliminate the tracking errors, and PID control can eliminate the synchronization errors.

The third term is the robust compensation term that can improve the system stability in the presence of disturbance and uncertainties [41]:

$$
\mathbf{u}_{3}=\mathbf{u}_{a u x},
$$

where $\mathbf{u}_{\text {aux }}$ denotes the auxiliary robust control law, and $\mathbf{u}_{\text {aux }}$ has three types of manner, as follows:

$$
\mathbf{u}_{\text {1aux }}=\rho \frac{\mathbf{S}}{\|\mathbf{S}\|},
$$

where $\rho$ is positive scalar, and $\mathbf{S} /\|\mathbf{S}\|$ is a symbolic function (i.e., sliding surface function) whose variation range is from -1 to 1 , so the system is also termed adaptive robust synchronous sliding mode control (AS-SMC).

$$
\mathbf{u}_{2 a u x}=\frac{1}{\delta} \rho^{2} \mathbf{S},
$$

where $\delta$ is positive scalar, and the smaller $\delta$ is, the bigger $\mathbf{u}_{2 a u x}$ is, so it is called adaptive robust synchronous high gain control, or AS-High Gain for short.

$$
\mathbf{u}_{3 a u x}=\frac{\rho^{2} \mathbf{S}}{\rho\|\mathbf{S}\|+\delta},
$$

$\mathbf{u}_{3 a u x}$ is compared with $\mathbf{u}_{1 a u x}$, if constant $\delta=0$, then $\mathbf{u}_{3 a u x}=\mathbf{u}_{1 \text { aux }}$. So $\mathbf{u}_{\text {laux }}$ is the special case of $\mathbf{u}_{3 a u x}$. If the constant $\delta>0$, then $\mathbf{u}_{3 a u x}$ compared with $\mathbf{u}_{1 a u x}$ illustrates that the former has a small fluctuation range, and more importantly its variation range is within -1 to 1 . Coincidentally, it also increases the frequency of vibration, so it is called adaptive robust synchronous high-frequency control or AS-High Frequency for short.

In a similar manner with (32), the following dynamic control equation can be written as a linear parameterization form with respect to these parameters:

$$
\mathbf{M} \ddot{\mathbf{X}}_{\mathrm{r}}+\mathbf{C} \dot{\mathbf{X}}_{\mathrm{r}}+\mathbf{G}=\mathbf{Y}\left(\dot{X}_{\mathrm{r}}, \ddot{X} \mathrm{r}, \dot{X}, \mathbf{X}\right) \Theta
$$

Then the linear parameterization of the dynamic equation enables us to derive

$$
\widehat{\mathbf{M}} \ddot{\mathbf{X}}_{\mathrm{r}}+\widehat{\mathbf{C}} \dot{\mathbf{X}}_{\mathrm{r}}+\widehat{\mathbf{G}}=\mathbf{Y}\left(\dot{X}_{\mathrm{r}}, \ddot{X}, \dot{\mathrm{X}}, \mathbf{X}\right) \widehat{\Theta}
$$

where $\widehat{\Theta}$ denotes the estimated values (time-varying) for the parameter vector $\Theta$, and the parameter estimation error $\widetilde{\Theta}$ can be described by

$$
\widetilde{\Theta}=\widehat{\Theta}-\Theta \text {. }
$$

Consequently, the control law can be also described with regression matrix, $\mathrm{i}$ e.,

$$
\mathbf{u}=\mathbf{Y}\left(\dot{\mathbf{X}}_{\mathrm{r}}, \ddot{\mathbf{X}}_{\mathrm{r}}, \dot{\mathbf{X}}, \mathbf{X}\right) \widehat{\Theta}+\mathbf{K S}+\mathbf{u}_{a u x}
$$

We now turn our attention to analyzing the stability of the closed system with the Lyapunov function candidate

$$
V(t)=\frac{1}{2} \mathbf{S}^{T} \mathbf{M S}+\frac{1}{2} \widetilde{\Theta}^{T} \Gamma^{-1} \widetilde{\Theta} .
$$

The differentiation of $V(t)$ with respect to time leads to

$$
\dot{V}(t)=\mathbf{S}^{\mathrm{T}}\left[\mathbf{M} \dot{\mathbf{S}}+\frac{1}{2} \dot{\mathbf{M} S}\right]+\widetilde{\Theta}^{\mathrm{T}} \Gamma^{-1} \dot{\widetilde{\Theta}} .
$$

Considering the relation in equations (41), (43), (53), and (54) yields

$$
\begin{aligned}
\mathbf{M} \ddot{\mathbf{X}}_{\mathrm{r}}+\mathbf{C} \dot{\mathbf{X}}_{\mathrm{r}}-(\mathbf{M} \ddot{\mathbf{X}}+\mathbf{C} \dot{\mathbf{X}}) & =\mathbf{M} \dot{\mathbf{s}}+\mathbf{C s} \\
& =\mathbf{Y}\left(\dot{\mathbf{X}}_{\mathrm{r}}, \ddot{\mathbf{X}}_{\mathrm{r}}, \dot{\mathbf{X}}, \mathbf{X}\right) \Theta-\mathbf{F}_{X} .
\end{aligned}
$$

So the time derivative of $V(t)$ can be further expressed as

$$
\begin{aligned}
\dot{V}(t) & =\mathbf{S}^{T}\left[\mathbf{M} \dot{\mathbf{S}}+\frac{1}{2} \dot{\mathbf{M}} \mathbf{S}\right]+\widetilde{\Theta}^{T} \Gamma^{-1} \dot{\widetilde{\Theta}} \\
& =\mathbf{S}^{T}\left[\mathbf{Y}\left(\dot{\mathbf{X}}_{\mathrm{r}}, \ddot{\mathbf{X}}, \dot{\mathbf{X}}, \mathbf{X}\right) \Theta-\mathbf{F}_{X}\right]+\frac{1}{2} \mathbf{S}^{T}(\dot{M}-2 \mathbf{C}) \mathbf{S}+\widetilde{\Theta}^{T} \Gamma^{-1} \dot{\widetilde{\Theta}} \\
& =\mathbf{S}^{T}\left[\mathbf{Y}\left(\dot{\mathbf{X}}_{\mathrm{r}}, \ddot{\mathbf{X}}, \dot{\mathbf{X}}, \mathbf{X}\right) \Theta-\mathbf{F}_{X}\right]+\widetilde{\Theta}^{T} \Gamma^{-1} \dot{\widetilde{\Theta}},
\end{aligned}
$$

where the skew symmetry of $\dot{\mathbf{M}}-2 \mathbf{C}$ can be utilized to eliminate the term $(1 / 2) \mathbf{S}^{T} \dot{M} \mathbf{S}$ which reflects the timevarying nature of the inertia matrix.

Substituting the control law (54) in the above equation, we obtain

$$
\dot{V}(t)=-\mathbf{S}^{T} \mathbf{K} \mathbf{S}-\mathbf{S}^{T} \mathbf{u}_{a u x}-\mathbf{S}^{T} \mathbf{Y}\left(\dot{\mathbf{X}}_{\mathrm{r}}, \ddot{\mathbf{X}}_{\mathrm{r}}, \dot{\mathbf{X}}, \mathbf{X}\right) \widetilde{\Theta}+\widetilde{\Theta}^{T} \Gamma^{-1} \dot{\widetilde{\Theta}} .
$$


It is worth noting that the following relation can be utilized:

$$
\left[\mathbf{S}^{T} \mathbf{Y}\left(\dot{\mathbf{X}}_{\mathrm{r}}, \ddot{\mathbf{X}}_{\mathrm{r}}, \dot{\mathbf{X}}, \mathbf{X}\right) \widetilde{\Theta}\right]^{T}=\mathbf{S}^{T} \mathbf{Y}\left(\dot{\mathbf{X}}_{\mathrm{r}}, \ddot{\mathbf{X}}_{\mathrm{r}}, \dot{\mathbf{X}}, \mathbf{X}\right) \widetilde{\Theta}
$$

The result can be further derived as

$$
\dot{V}(t)=-\mathbf{S}^{T} \mathbf{K S}-\mathbf{S}^{T} \mathbf{u}_{a u x}-\widetilde{\Theta}^{T}\left[\mathbf{Y}\left(\dot{\mathbf{X}}_{\mathrm{r}}, \ddot{\mathbf{X}}_{\mathrm{r}}, \dot{\mathbf{X}}, \mathbf{X}\right)^{T} \mathbf{s}-\Gamma^{-1} \dot{\widetilde{\Theta}}\right] .
$$

Let $\mathbf{Y}\left(\dot{\mathbf{X}}_{\mathrm{r}}, \ddot{\mathbf{X}}_{\mathrm{r}}, \dot{\mathbf{X}}, \mathbf{X}\right)^{T} \mathbf{S}-\mathbf{S}^{T} \mathbf{u}_{a u x}-\Gamma^{-1} \dot{\widetilde{\Theta}}=0$ satisfy.

Then, one can obtain

$$
\dot{\widetilde{\Theta}}=\Gamma \mathbf{Y}\left(\dot{\mathbf{X}}_{\mathrm{r}}, \ddot{\mathbf{X}}_{\mathrm{r}}, \dot{\mathbf{X}}, \mathbf{X}\right)^{T} \mathbf{S} .
$$

The adaptive law, i.e., the unknown dynamic parameters, can be estimated with

$$
\dot{\widehat{\Theta}}=\dot{\widetilde{\Theta}}=\Gamma \mathbf{Y}\left(\dot{\mathbf{X}}_{\mathrm{r}}, \ddot{\mathbf{X}}_{\mathrm{r}}, \dot{\mathbf{X}}, \mathbf{X}\right)^{T} \mathbf{S} .
$$

Equation (63) is finally reduced to

$$
\dot{V}(t)=-\mathbf{S}^{T} \mathbf{K S}-\mathbf{S}^{T} \mathbf{u}_{a u x} \leq 0 .
$$

As $\mathbf{K}$ is a positive definite matrix, so $\dot{V}(\mathbf{0})=0$ and $\dot{V}(t) \leq 0$ which is proved to be a seminegative definite matrix. The sliding surface $\mathbf{S}$ will asymptotically converge to zero, $t \longrightarrow \infty$, and $\mathbf{S}=\mathbf{0}$. From equations (39) and (40), we can see that $\mathbf{e}^{*}$ will be asymptotically zero, $\dot{\mathbf{e}}^{*}$ also converges to zero. Furthermore, from equation (37), $\boldsymbol{\varepsilon}, \dot{\boldsymbol{\varepsilon}}$, e, and $\dot{\mathbf{e}}$ will converge to zero, and the upper platform can asymptotically track a desired trajectory in the Cartesian space, i.e., $\mathbf{X} \longrightarrow \mathbf{X}_{d}$, the closed-loop system will be globally stable within finite time.

It is worth noting that the involved manipulator is a redundantly actuated parallel manipulator, so the driving force is redundant, which leads to multiple solutions of the input driving force. In order to reduce the internal force and improve the system control accuracy, the driving force is required to optimize and choose the optimal solution from multiple solutions. Herein, aiming at the minimum driving force value and reducing the internal force of the manipulator, the Euclidean norm of the driving force is selected to optimize the driving force. The optimized objective function can be expressed as

$$
f=\tau_{\mathrm{a}}^{T} \mathbb{N} \tau_{\mathrm{a}},
$$

where $\tau_{\mathrm{a}}$ is the driving forces vector exerted on the actuated joints, and $\mathbb{N}$ is the harmonious matrix of the actuator motors.

The optimization is constrained by the condition that linear mapping relationship between the actuated joint force and corresponding Cartesian space force, namely,

$$
\mathbf{F}_{X}=\mathbf{J}_{0}^{T} \boldsymbol{\tau}_{\mathrm{a}}
$$

The equation can be constructed by the Lagrange equation:

$$
\bar{f}=\boldsymbol{\tau}_{\mathrm{a}}^{T} \mathbb{N} \boldsymbol{\tau}_{\mathrm{a}}+\lambda\left(\mathbf{F}_{X}-\mathbf{J}_{0}^{T} \boldsymbol{\tau}_{\mathrm{a}}\right)
$$

Partial derivative of the abovementioned equation (77) yields

$$
\left\{\begin{array}{l}
\frac{\partial \bar{f}}{\partial \boldsymbol{\tau}_{\mathrm{a}}}=2 \boldsymbol{\tau}_{\mathrm{a}} \mathbb{N}-\lambda \mathbf{J}_{0}^{T}, \\
\frac{\partial \bar{f}}{\partial \lambda}=\mathbf{F}_{X}-\mathbf{J}_{0}^{T} \boldsymbol{\tau}_{\mathrm{a}} .
\end{array}\right.
$$

Let the partial derivative be equal to zero and find the extreme value

$$
\left\{\begin{array}{l}
\boldsymbol{\tau}_{\mathrm{a}}=\frac{1}{2} \mathbb{N}^{-T} \mathbf{J}_{0} \lambda^{T}, \\
\mathbf{F}_{X}=\mathbf{J}_{0}^{T} \boldsymbol{\tau}_{\mathrm{a}} .
\end{array}\right.
$$

Combining the above two equations in (71) leads to

$$
\mathbf{F}_{X}=\frac{1}{2} \mathbf{J}_{0}^{T} \mathbb{N}^{-T} \mathbf{J}_{0} \lambda^{T}
$$

One can express with another form as well, namely,

$$
\lambda^{T}=2\left(\mathbf{J}_{0}^{T} \mathbb{N}^{-T} \mathbf{J}_{0}\right)^{-1} \mathbf{F}_{X}
$$

Substituting equation (73) in the first equation of (71), one can obtain the optimal driving force:

$$
\boldsymbol{\tau}_{\mathrm{a}}=\mathbb{N}^{-T} \mathbf{J}_{0}\left(\mathbf{J}_{0}^{T} \mathbb{N}^{-T} \mathbf{J}_{0}\right)^{-1} \mathbf{F}_{X}=\mathbf{J}_{0}\left(\mathbf{J}_{0}^{T} \mathbf{J}_{0}\right)^{-1} \mathbf{F}_{X}
$$

Therefore, the control law in actuated joints space can be described as

$$
\boldsymbol{\tau}_{\mathrm{a}}=\mathbf{J}_{0}\left(\mathbf{J}_{0}^{T} \mathbf{J}_{0}\right)^{-1} \mathbf{F}_{X}=\mathbf{J}_{0}\left(\mathbf{J}_{0}^{T} \mathbf{J}_{0}\right)^{-1} \mathbf{u} .
$$

Finally, the overall diagram of the proposed control scheme is described in Figure 4, from which one can see that the control scheme consists of three main parts.

The desired trajectory parameter $\mathbf{X}_{d}$ can be obtained once the trajectory function is given, while the actual operational task space parameter $\mathbf{X}$ can be obtained with two methods. One is forward kinematics in which the position of the actuated joints are once known, then the position and orientations of the moving platform can be obtained by adopting the back propagation (BP) neural network optimization method or Newton-Raphson iteration, which converges rather quickly when the initial value is close to the desired solution [42]. However, the solving procedure of forward kinematics is very complicated and the computational efficiency is very low. The other efficient method to require the position and orientations of the moving platform is exactly what we want to introduce in this paper [43-45]. We know that the position $z$ of the moving platform can be straightforwardly obtained by the position sensor, yet the rotational Euler angels cannot be required easily, maybe the angular velocity can be obtained by gyroscope sensor, but it is of no use in calculation. The rotation matrix $\mathbf{R}$ defining the orientation of the moving platform with respect to the fixed coordinate system can be availably obtained by the camera sensor technique. We can obtain the rotational Euler angles $\alpha$ and $\beta$ by interfacing with MATLAB Stereo Camera 


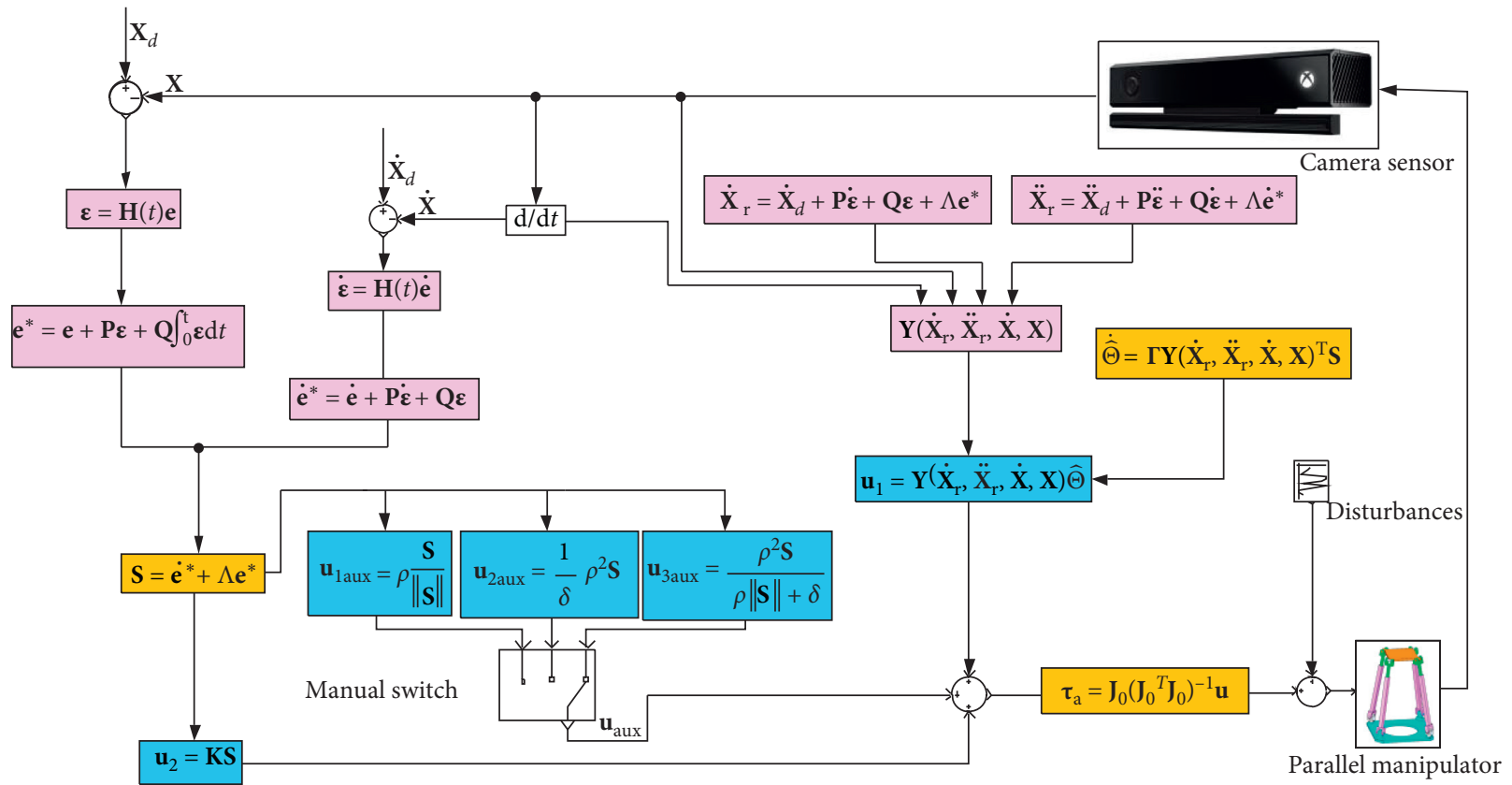

FIGURE 4: The proposed three adaptive robust synchronous control diagram of parallel manipulator.

Calibrator Toolbox [46] with equation (2), and the position distance can be figured out in real time on the personal computer. It is computationally simple, and it does not require measurement of the end-effector velocity and acceleration as well. The advantage of proposed simplified method can efficiently avoid the complex forward kinematics.

\section{Simulation Analysis Tests}

The parallel manipulator system in real environment is fabricated as shown in Figure 5, which mainly consists of a parallel manipulator, a U2D (or USB2Dynamicel, which is a small size USB communication converter that enables to control and to operate the Dynamixel with the personal computer) controller, a personal computer and a binocular camera (herein, binocular stereo vision technology are not discussed temporarily). The parallel manipulator can move by changing the rotation angles of four smart actuators (Dynamixel MX-12W, which is a high-performance actuator with a fully integrated DC motor, reduction gearhead, controller, driver, and network, all in one servo module actuator). The real position and velocity of the four actuators will be transmitted to the computer for data monitoring and implementing control in real time.

The Dynamixel actuator needs to be calibrated before the experiment and connected the personal computer through U2D (or USB2Dynamixel). The actuator information can be modified in terms of the software RoboPlus, and the information can be updated in Dynamixel Wizard modular, as shown in Figure 6. Some more information can be obtained on the ROBOTIS e-Manual [47]. In addition, the camera also needs to be calibrated with a square chessboard; when a sequence of different chessboard images are given, we can obtain the camera information (such as camera position and camera matrix) so as to detect the position and orientation of the parallel manipulator. More information has been addressed in [48] where the authors proposed an automatic calibration algorithm by virtue of the two Hough transform, image corners, and invariant properties of the perspective transformation to determine the calibration points from a sequence of different images, and herein we do not discuss this issue in detail.

However, to validate the performance of the proposed controllers, first of all, the control schemes for the parallel manipulator are implemented within SimMechanics Toolbox environment, and the experimental tests will be executed in next paper. The simplified structural parameters of the 2RPU-2SPR parallel manipulator are listed in Table 1. Meanwhile, the constant parameters implemented in the three robust controllers are presented in Table 2.

To show the performance of the three robust controllers proposed in this paper, a challenging reference trajectory was developed. Specifically, the desired trajectory function of the moving platform generated by the following equations:

$$
\left\{\begin{array}{l}
\alpha(t)=\frac{\pi}{6} \sin (2 t), \\
\beta(t)=\frac{\pi}{6} \cos (2 t), \\
z(t)=0.5+0.04 t .
\end{array}\right.
$$

The initial position and orientation of the moving platform is $\left[\begin{array}{lll}z & \alpha & \beta\end{array}\right]^{T}=\left[\begin{array}{lll}0.7 & 0 & 0\end{array}\right]^{T}$, the simulation time is 8 seconds, the sampling time of the control system is 1 millisecond, and the solver is ode4 (Runge-Kutta) in Simulink, respectively.

The comparative experiments are carried out for the above three controllers. Figure 7 illustrates the desired 


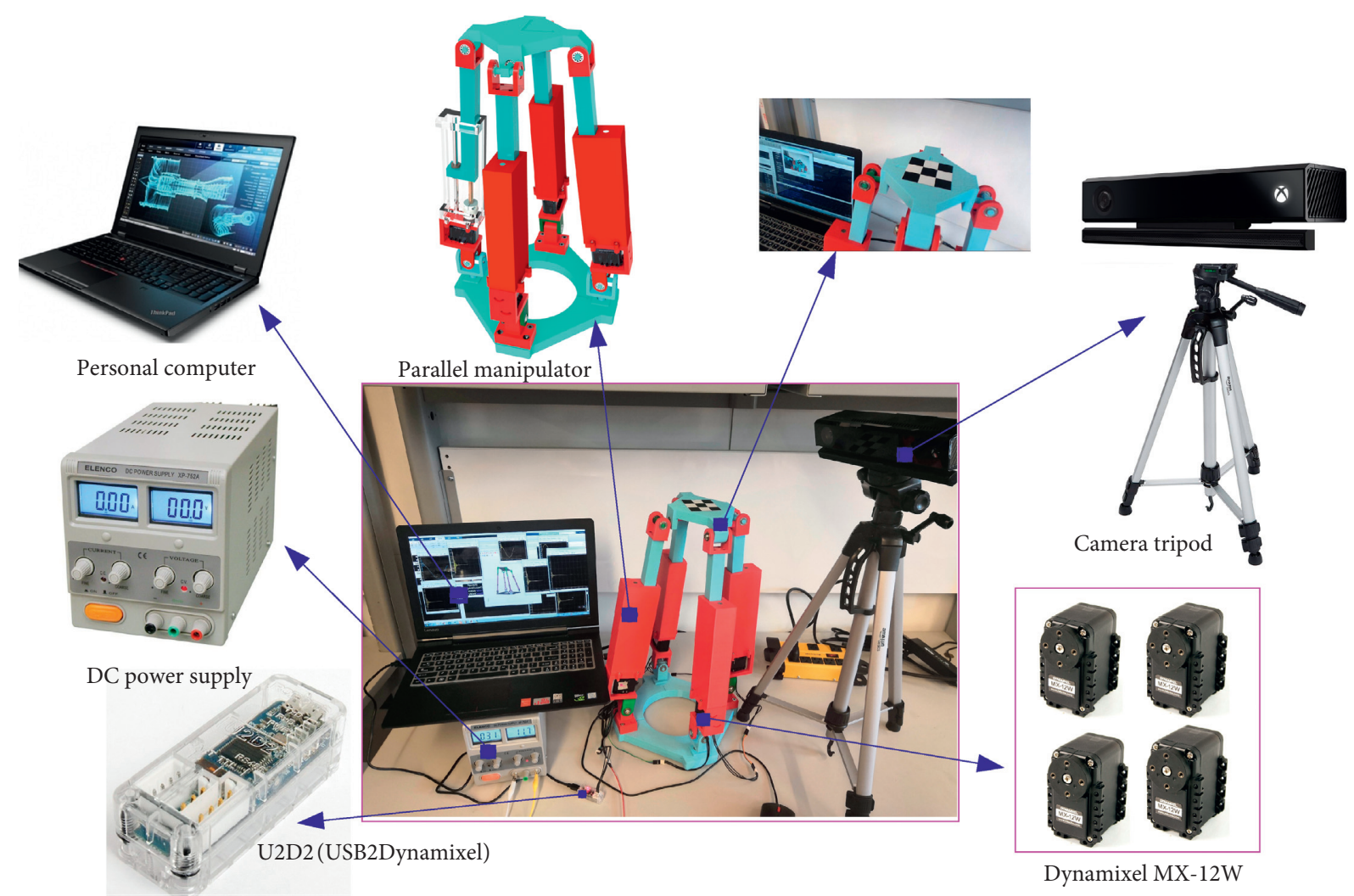

Figure 5: The simulation and experimental platform of the parallel manipulator.

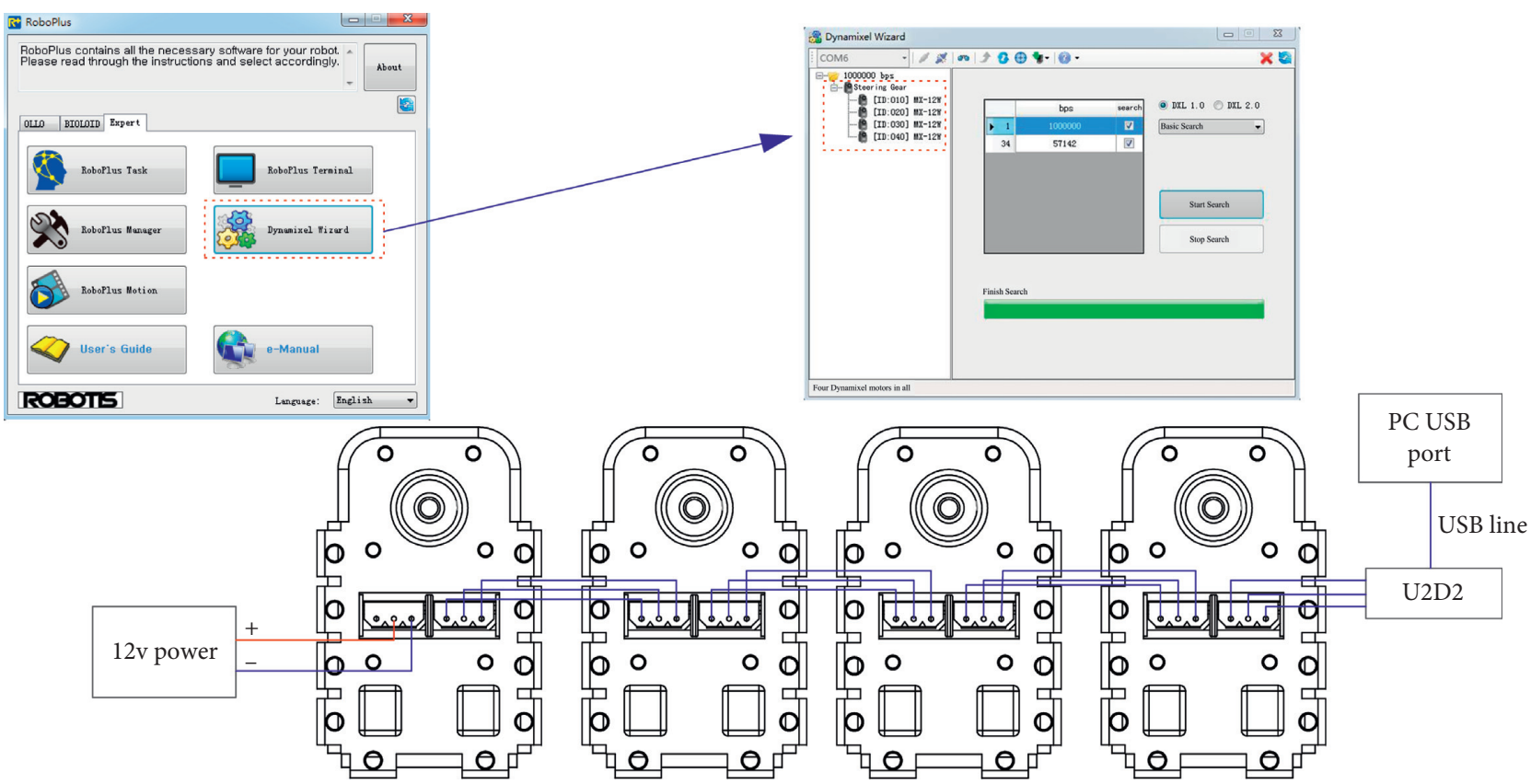

FIgURE 6: The schematic diagram of Dynamixel actuator calibration.

trajectory in the Cartesian space, the black dot indicates the starting point, and the desired trajectories of three degrees of freedom in the Cartesian space are depicted in Figure 8, respectively. From these results, it can be seen that the proposed three controllers can make the tracking error for the desired trajectory globally asymptotically stable and achieve good trajectory tracking performance in the Cartesian space, since the unknown parameters can be accurately estimated by the adaptive law and then compensate with the PID and PD feed-forward linear control method. 
TABLE 1: Structural parameters of the parallel manipulator.

\begin{tabular}{lcc}
\hline Parameter & Description & Value \\
\hline$r_{a}$ & The radius of the moving platform & $0.2 \mathrm{~m}$ \\
$r_{b}$ & The radius of the fixed platform & $0.339 \mathrm{~m}$ \\
$m_{a}$ & The mass of the moving platform & $12.75 \mathrm{~kg}$ \\
$e_{1}$ & The distance between centroid and $B_{i}$ joint & $0.23 \mathrm{~m}$ \\
$e_{2}$ & The distance between centroid and $A_{i}$ joint & $0.22 \mathrm{~m}$ \\
$I_{p}$ & The inertia matrix of moving platform & $0.1 \times \operatorname{diag}\{0.77,1.07,1.83\} \mathrm{kg} * \mathrm{~m}^{2}$ \\
$m_{1}$ & The mass of the lower sublimb & $0.95 \mathrm{~kg}$ \\
$I_{1}$ & The inertia matrix of the lower sublimb & $0.01 \times \operatorname{diag}\{2.88,2.89,0.05\} \mathrm{kg} * \mathrm{~m}^{2}$ \\
$m_{2}$ & The mass of the upper sublimb & $1.1 \mathrm{~kg}$ \\
$I_{2}$ & The inertia matrix of the upper sublimb & $0.01 \times \operatorname{diag}\{2.65,2.67,0.03\} \mathrm{kg} * \mathrm{~m}^{2}$ \\
$g$ & The gravity acceleration & {$[0,0,-9.81]^{T} \mathrm{~m} / \mathrm{s}^{2}$} \\
\hline
\end{tabular}

TABLE 2: Control gains of the utilized controllers.

\begin{tabular}{lc}
\hline Parameter & Value \\
\hline $\mathbf{P}$ & $\operatorname{diag}\{0.3,0.3,0.3\}$ \\
$\mathbf{Q}$ & $\operatorname{diag}\{0.5,0.5,0.5\}$ \\
$\mathbf{K}$ & $\operatorname{diag}\{600,300,300\}$ \\
$\Lambda$ & $\operatorname{diag}\{100,50,50\}$ \\
$\rho$ & 10 \\
$\delta$ & 0.5 \\
\hline
\end{tabular}

The tracking errors in the Cartesian space of three controllers are presented in Figure 9. The position errors and orientation errors are drawn respectively, and the proposed controllers applied to the parallel manipulator for the prescribed trajectory tracking control are available. When the control system reaches a steady state, the errors will decrease gradually to zero, although the errors oscillated at the beginning or within some certain time. It is worth noting that AS-SMC and AS-High Frequency have better tracking performance than the AS-High Gain, especially on the $z$ axis, i.e., the tracking error $e_{1}$. Furthermore, AS-SMC has an equivalent or approximate equivalent tracking performance with AS-High Frequency.

In addition, the synchronous errors in the Cartesian space of three controllers are presented in Figure 10. By comparing the synchronous tracking performance $\varepsilon_{1}$ and $\varepsilon_{3}$, we find that three controllers have approximate equivalent variation period and sustained time. But from the synchronous tracking error $\varepsilon_{2}$, it can be seen that the AS-High Gain has great effect on the orientation tracking error in the Cartesian space but increase the synchronization error significantly. That is to say, AS-High Gain relies on high gain to adjust the error to achieve the goal of tracking.

The cross-coupling errors of three controllers are depicted in Figure 11. It is interesting to note that the influence laws are similar to trajectory tracking errors. In Figure 11, it is also clear that the change of the crosscoupling errors always causes a sharp increase before the cross-coupling errors converge to a very small value or less than a small value closer to zero. The developed AS-SMC and AS-High Frequency controllers show remarkable crosscoupling performances superior to AS-High Gain control scheme in three-degree-of-freedom directions.

The trajectory tracking errors are not only of importance in Cartesian space, but also important in joint space. The tracking errors in joint space are illustrated in Figure 12, which shows the experimental trajectory tracking results in each prismatic joint for all the three controllers. We hope that all the actuated joints can be controlled to move in a synchronous way. Otherwise, a sudden impact may occur to damage the mechanical structures. In Figure 12, we can see that the errors of the actuated joints are very small, and the variation mainly appears within 0.5 seconds. In the next 7.5 seconds, the errors almost approach zero approximately, which demonstrates that the designed controllers have the advantage of quick response speed and excellent trajectory tracking results. In addition, the difference between AS-SMC and AS-High Frequency controllers is almost imperceptible, and the AS-High Gain controller is restricted to a large vicinity of the zero. All the tracking errors were limited to a small region with deviation from zero. The tracking errors are not leaving the zone as shown in Figure 12.

Similarly, the force tracking errors are very important to validate the controller performance and the simulation results including four actuators in joint space are illustrated in Figure 13. We should notice that the rand disturbances (for example, frictions, noises, clearances, wears, and so on.) are considered in the simulation process. Herein, we assume that the lumped uncertainty obeys discrete uniform random distribution, i.e.,

$$
\boldsymbol{\tau}_{\mathrm{d}}^{\prime} \in \text { unifrnd (mu, sigma), }
$$

where unifrnd random obeys the discrete uniform distribution, mu is the minimum value, and sigma is the maximum value. Herein, we define $m u=-5$, sigma $=5$.

Further, we can see that the force tracking errors deviate from zero dramatically and they are driven back to the steady state fast after 0.5 seconds. However, the errors have a large fluctuation within 0.5 seconds, especially in the third picture with the AS-High Gain controller. The unexpected dash may destroy the structural components, so we should improve significantly and pay attention to this problem in the experimental process. In addition, it can be seen that the ASHigh Frequency controller achieves the best force tracking performance from the experimental results. The tracking errors are relatively stable, since it introduces high-frequency robust technique into the controller design. The ASHigh Frequency controller has better effectiveness compared with other controllers to guarantee stability in presence of unknown disturbance. 


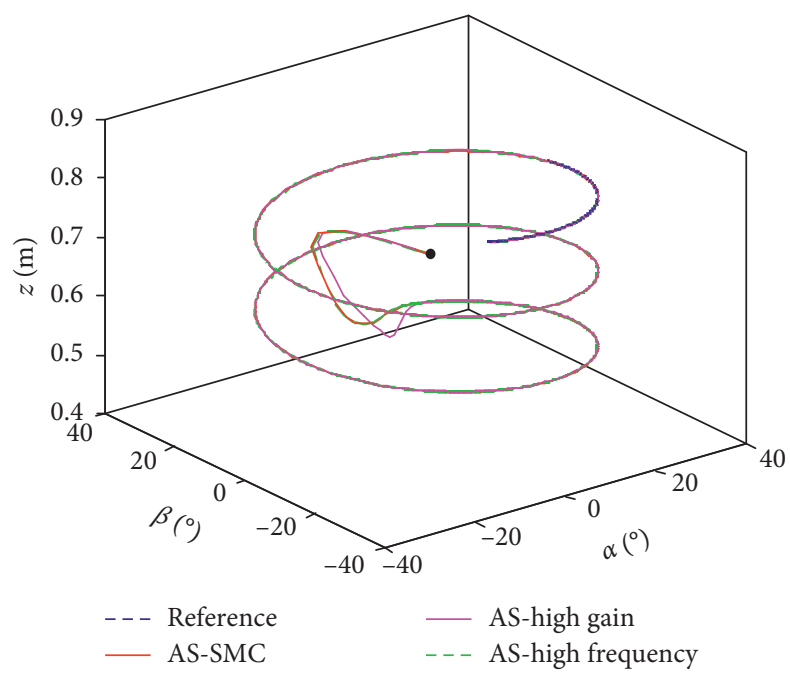

Figure 7: Desired trajectory in Cartesian space.

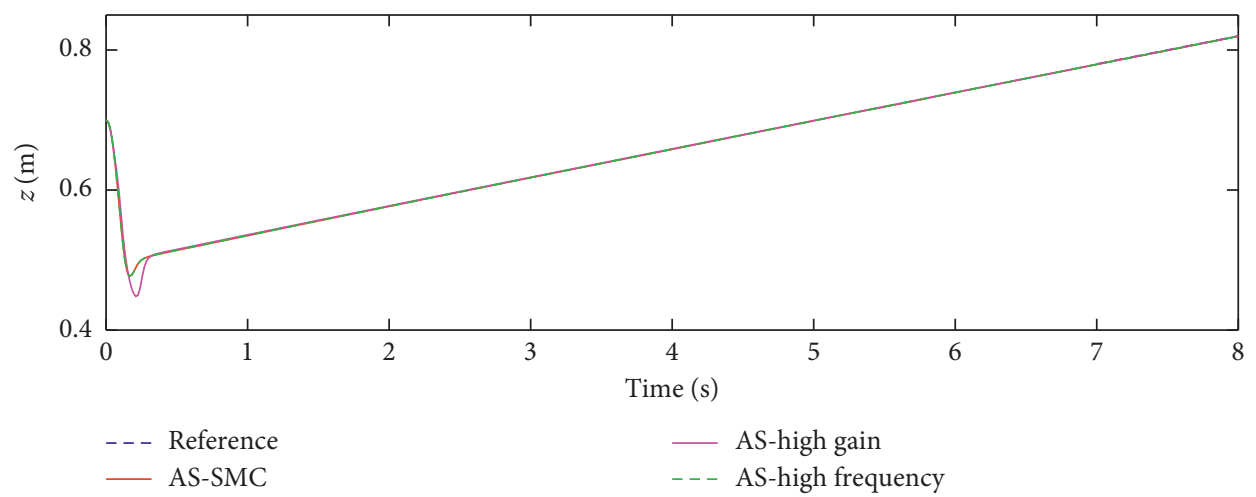

(a)

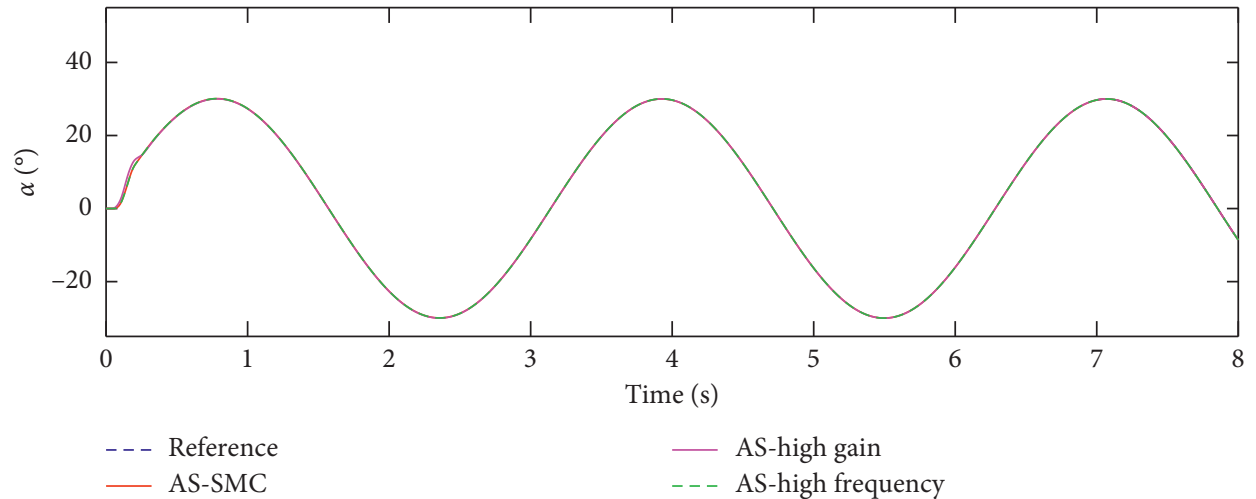

(b)

Figure 8: Continued. 


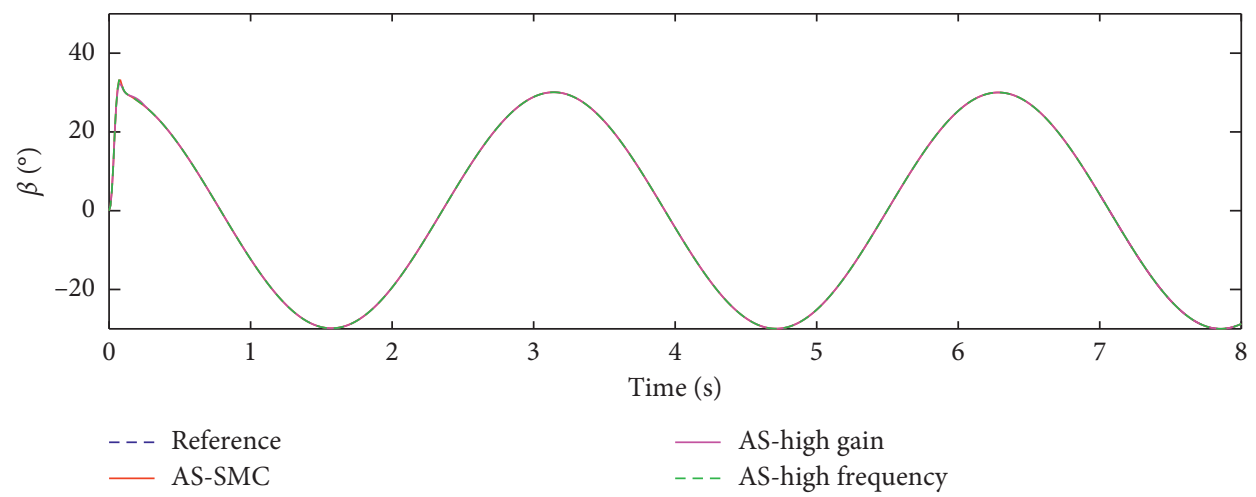

(c)

FIGURE 8: Desired trajectory of three degrees of freedom.

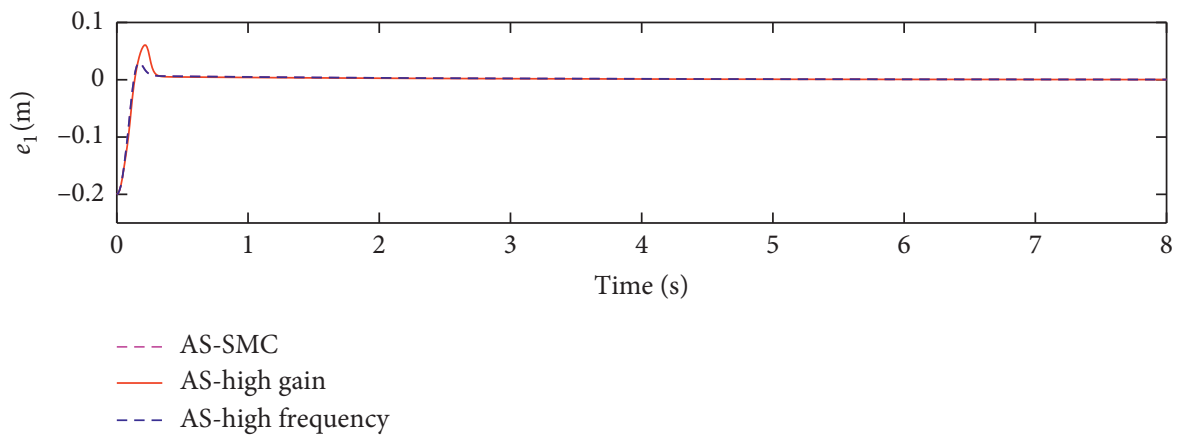

(a)

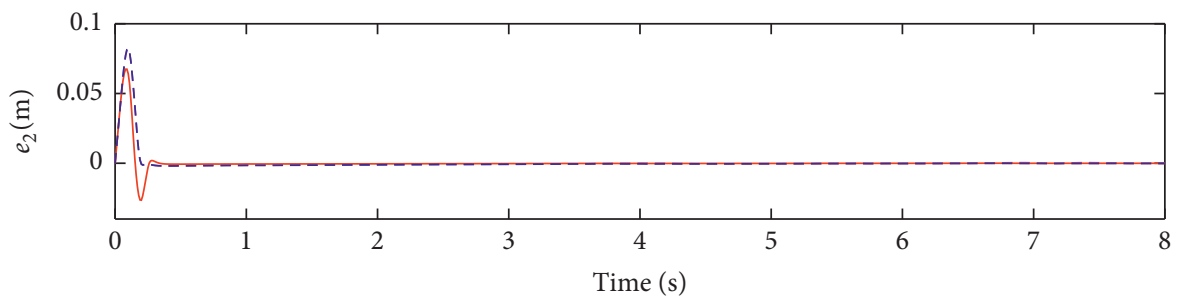

- - AS-SMC

— AS-high gain

- - - AS-high frequency

(b)

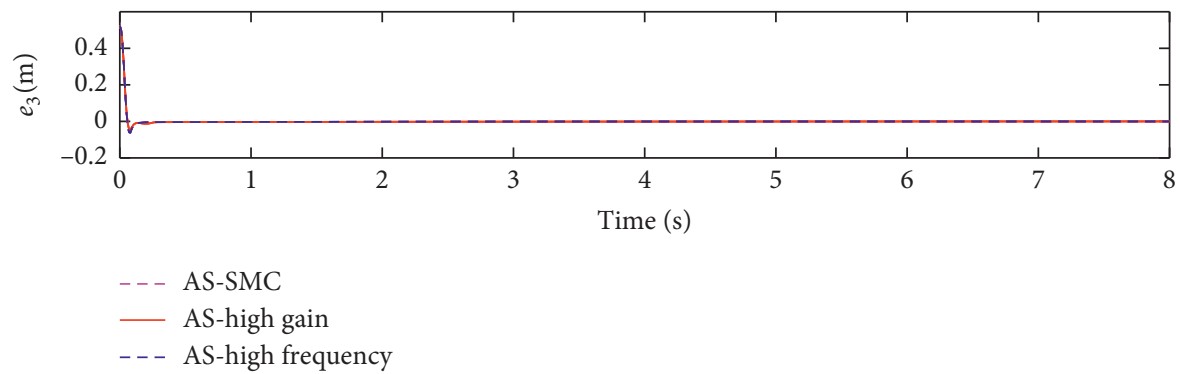

(c)

FIgURE 9: The trajectory tracking error.

A closer view to the force tracking errors in joint space is shown in Figure 14, which demonstrates that the AS-High Frequency controller approaches zero more accurately than the two other controllers, especially in the fourth picture. In general, the steady state is relative, and the errors still display small chattering. Furthermore, the magnitude of the chattering in the error state is directly related to the disturbance terms. Moreover, the AS-High Gain controller has a high 


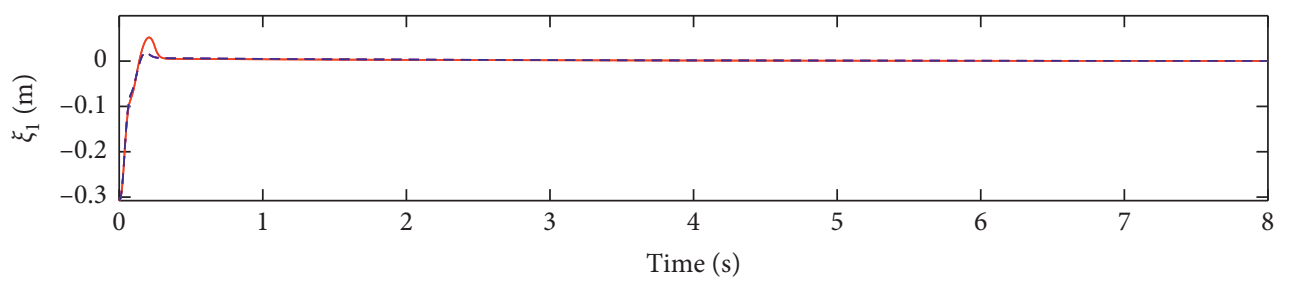

-- - AS-SMC

AS-high gain

- - - AS-high frequency

(a)

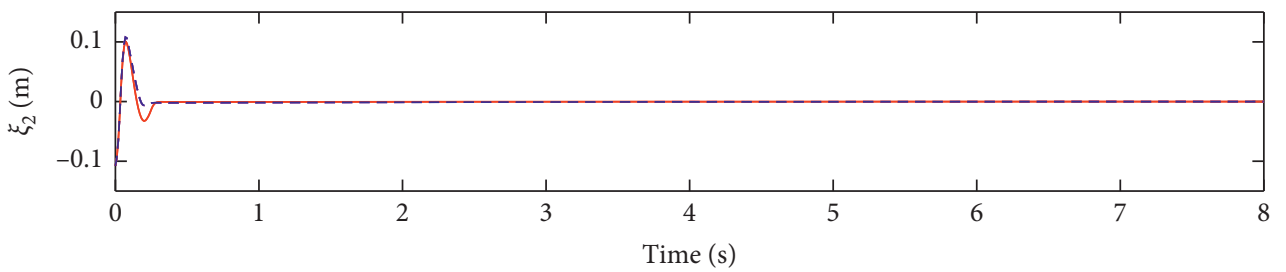

-.- AS-SMC

— AS-high gain

- - - AS-high frequency

(b)

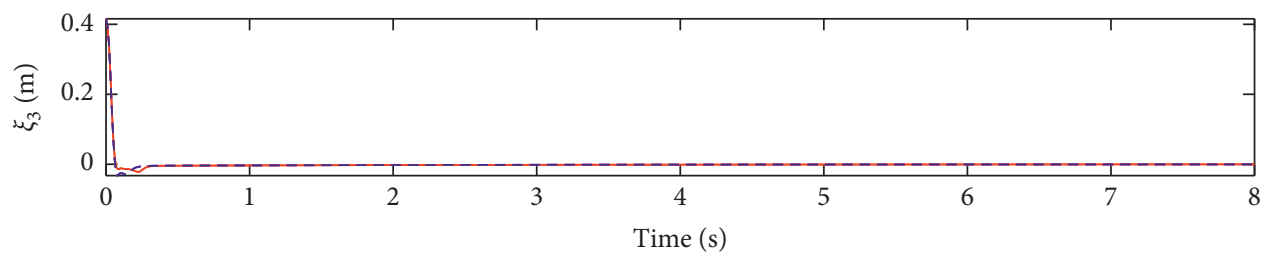

AS-SMC

AS-high gain

- - - AS-high frequency

(c)

FIgURE 10: The synchronization errors.

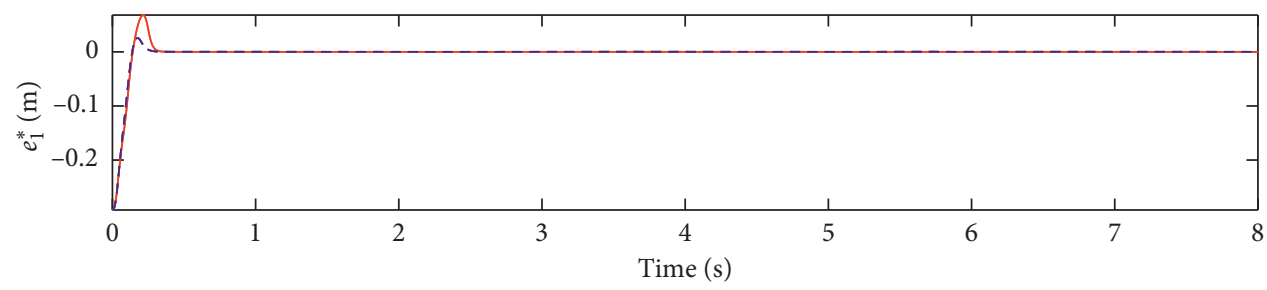

-.- AS-SMC

— AS-high gain

- - - AS-high frequency

(a)

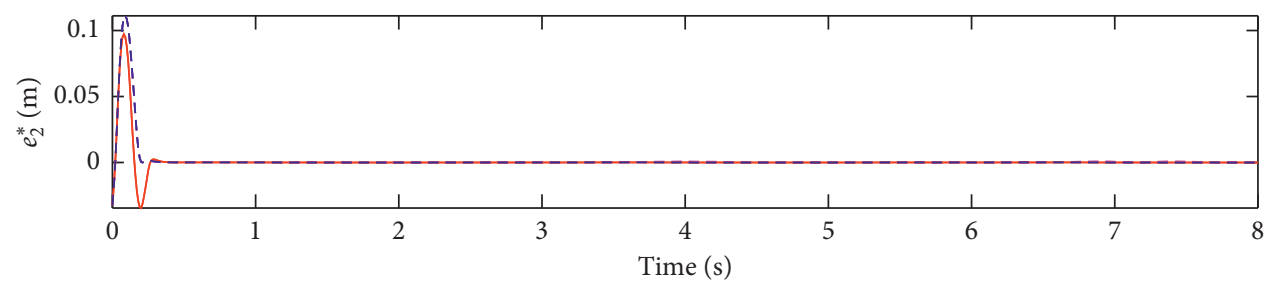

- - AS-SMC

— AS-high gain

- - - AS-high frequency

(b)

Figure 11: Continued. 


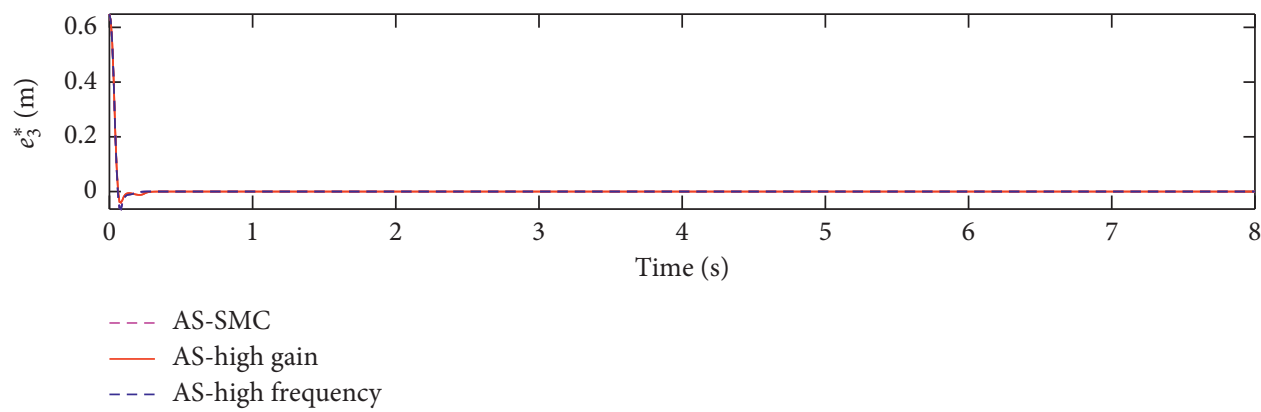

(c)

Figure 11: The cross-coupling errors.

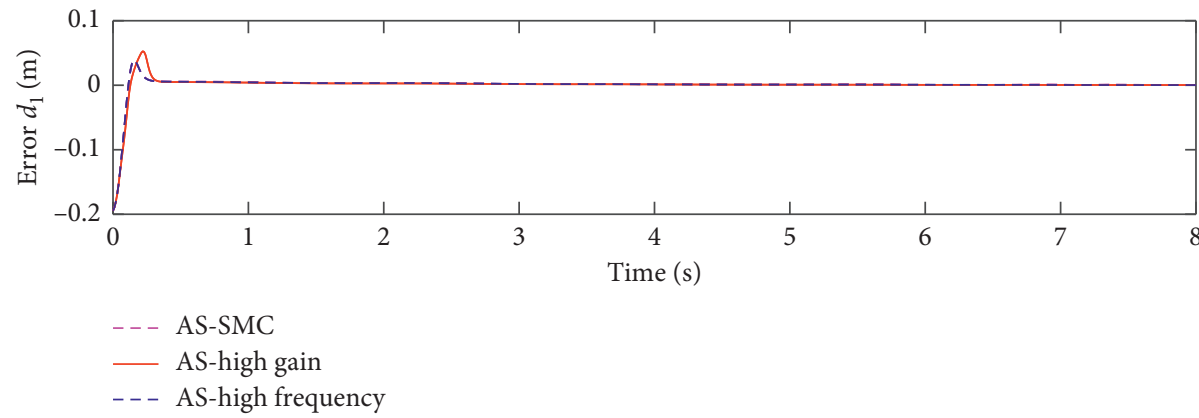

(a)

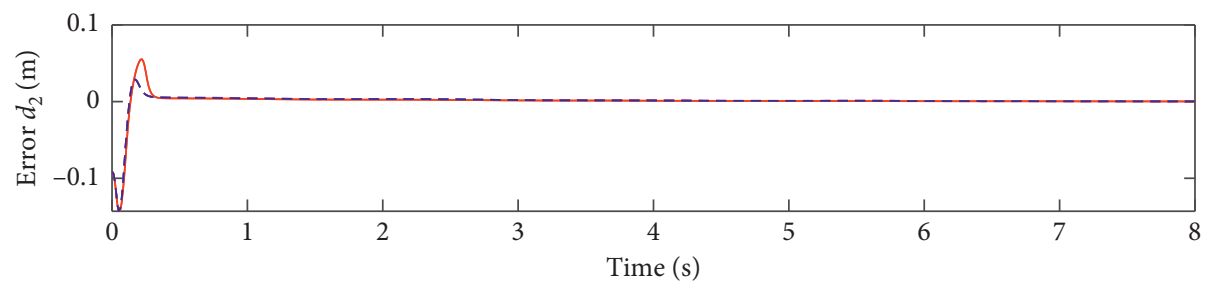

-. - AS-SMC

_ AS-high gain

- - - AS-high frequency

(b)

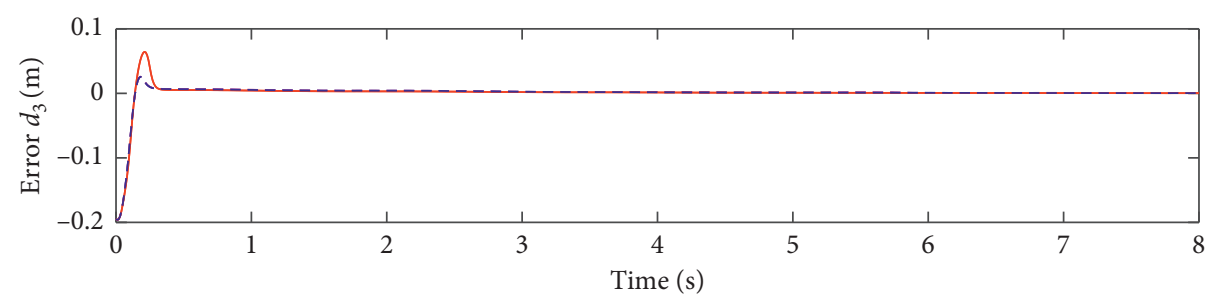

- - AS-SMC

— AS-high gain

- - - AS-high frequency

(c)

Figure 12: Continued. 


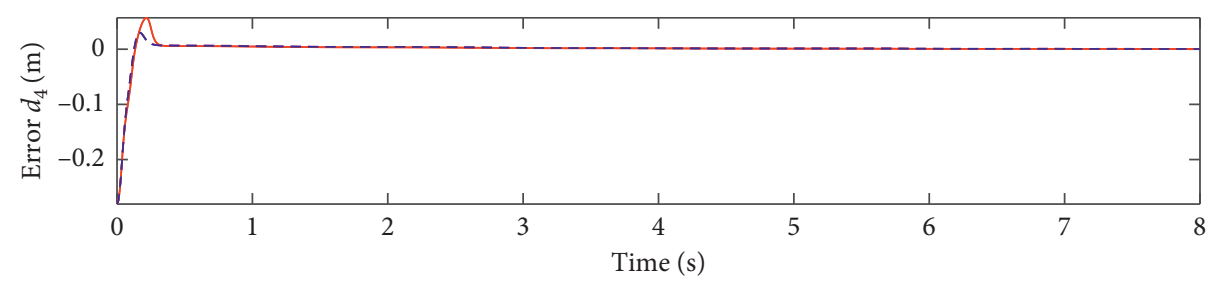

- - - AS-SMC

_ AS-high gain

- - - AS-high frequency

(d)

FIgURE 12: The position errors of actuator joints.

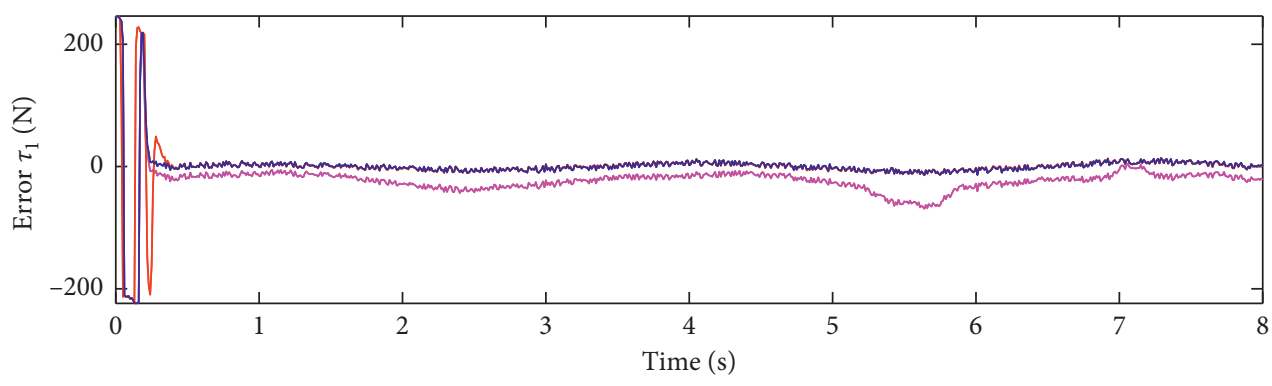

- - AS-SMC

_ AS-high gain

- - - AS-high frequency

(a)

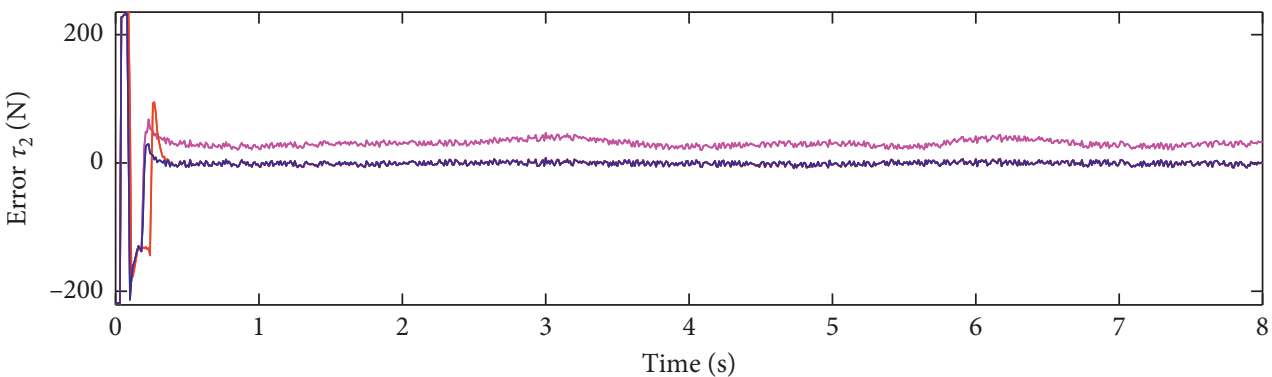

-.- AS-SMC

_ AS-high gain

- - - AS-high frequency

(b)

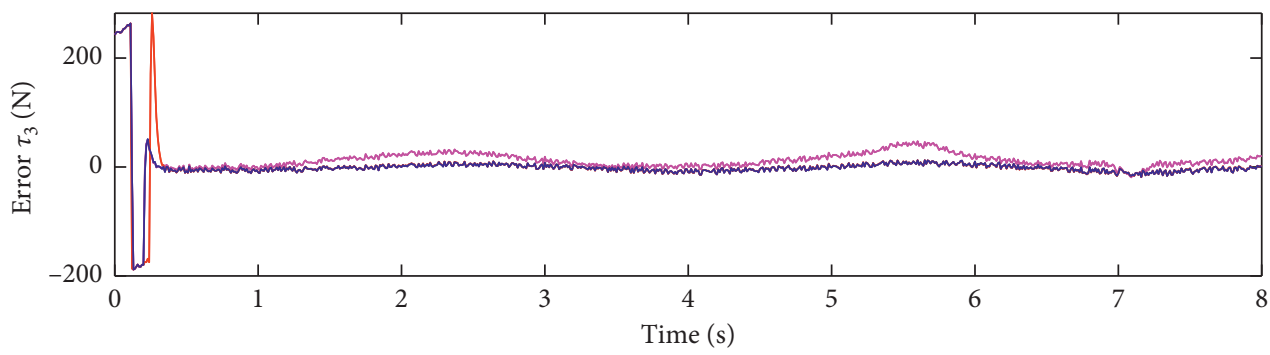

- - - AS-SMC

— AS-high gain

- - - AS-high frequency

(c)

Figure 13: Continued. 


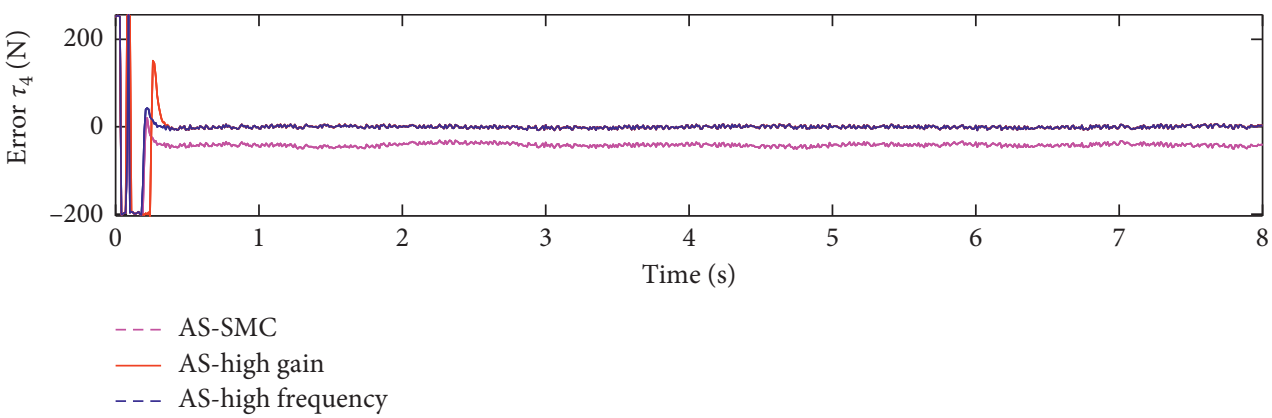

(d)

Figure 13: The force errors of actuator joints.

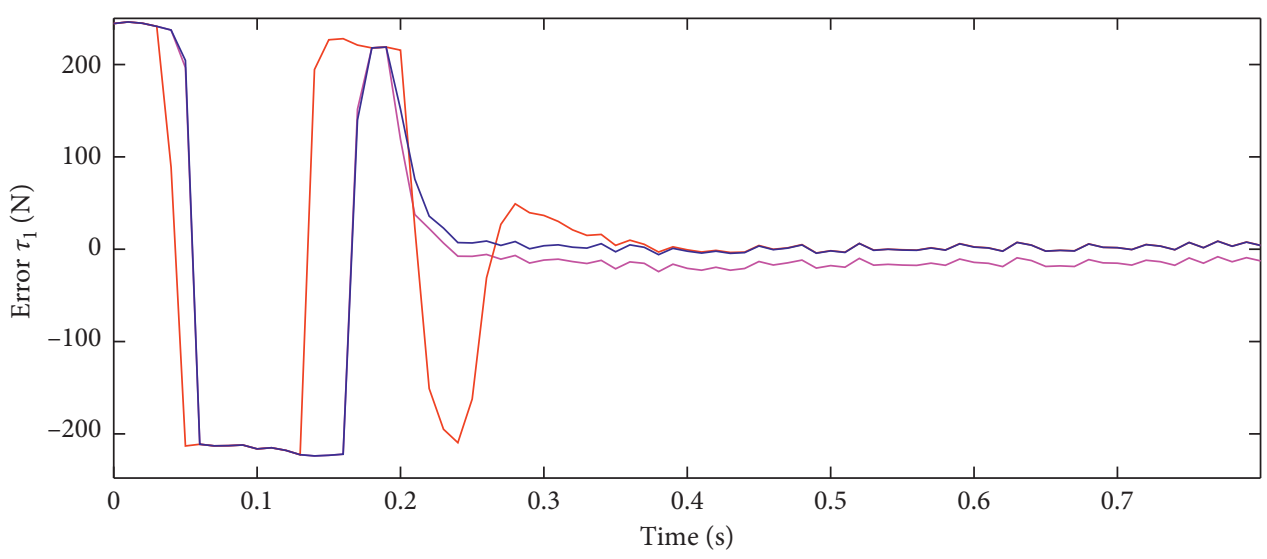

- AS-SMC

- AS-high gain

_ AS-high frequency

(a)

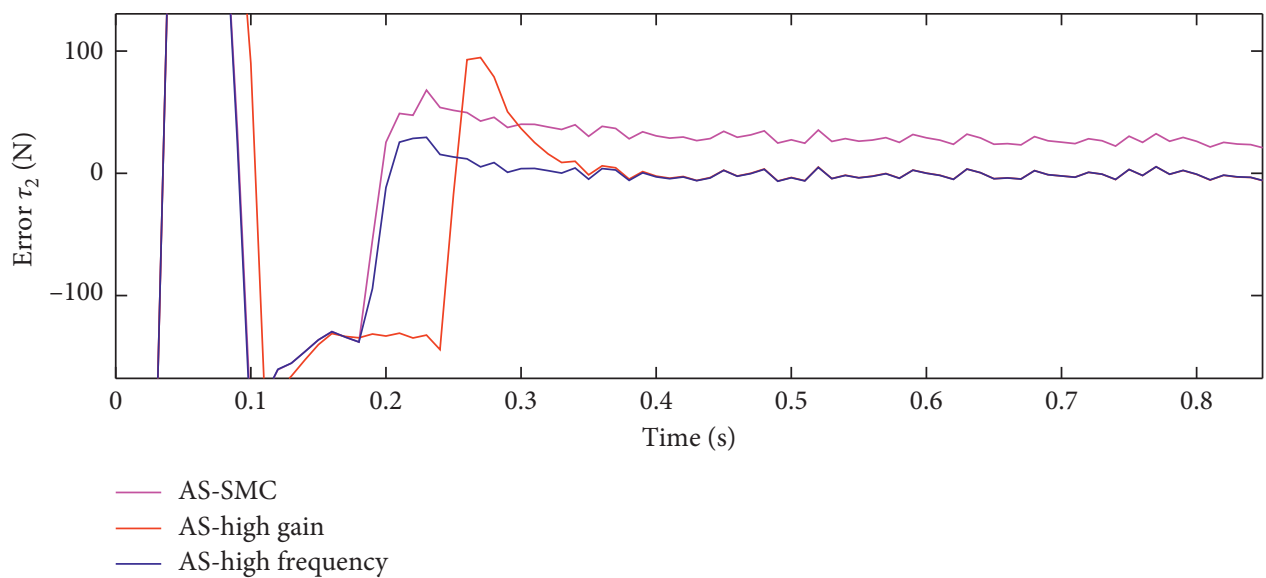

(b)

Figure 14: Continued. 


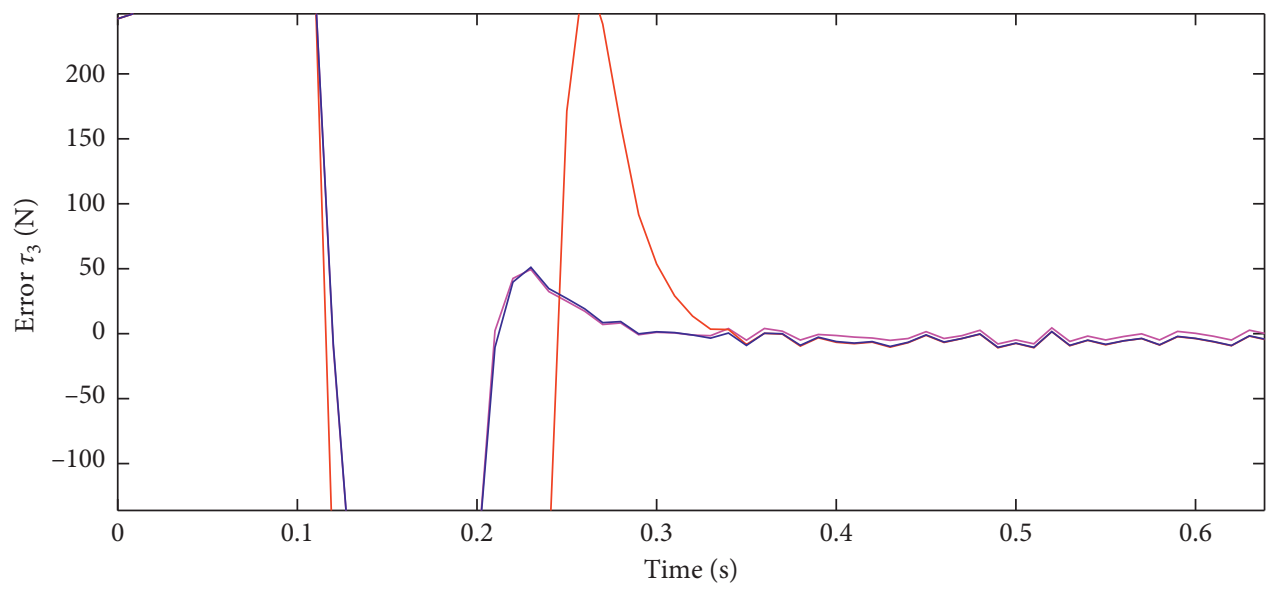

- AS-SMC

AS-high gain

— AS-high frequency

(c)

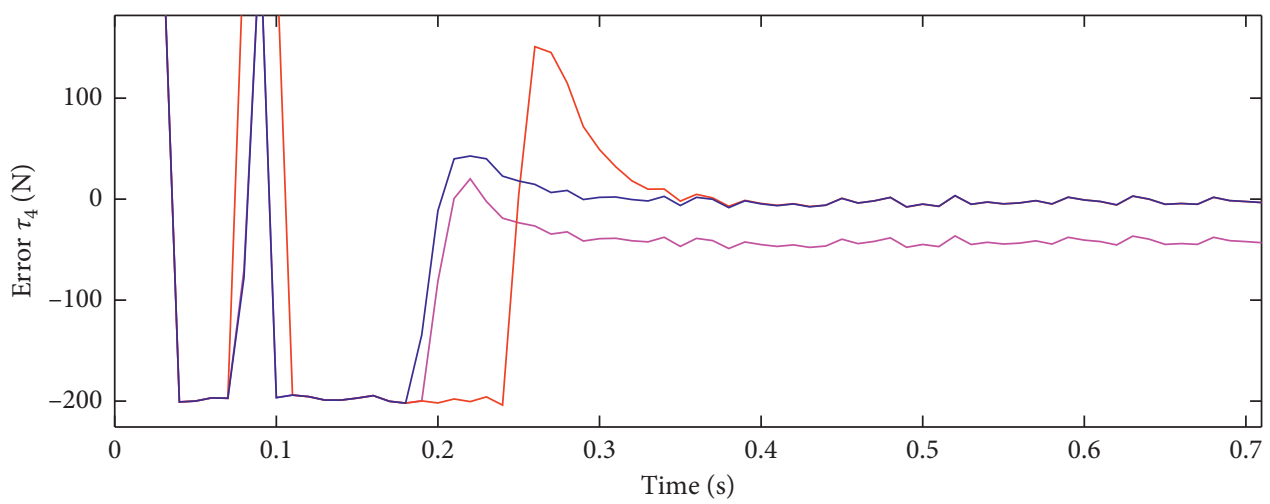

- AS-SMC

AS-high gain

AS-high frequency

(d)

Figure 14: The force errors of partial enlargement.

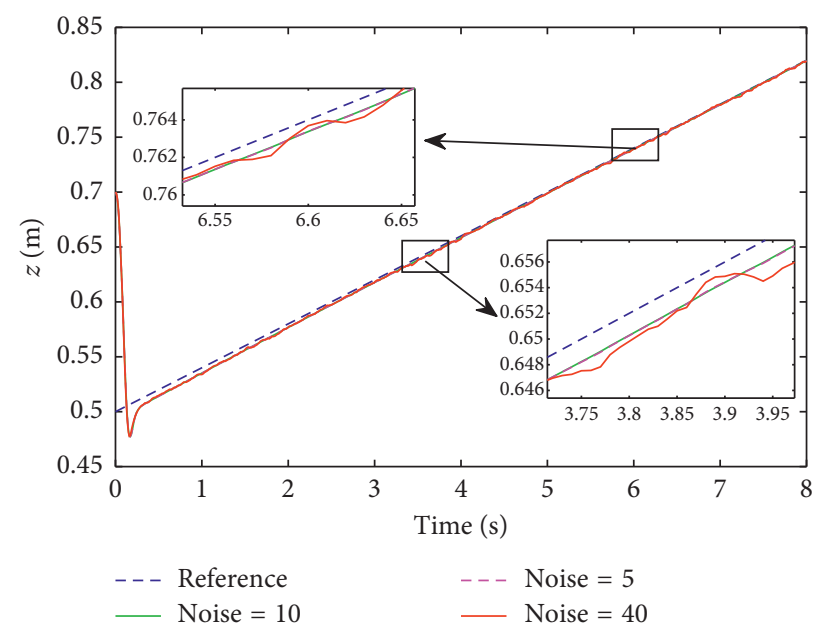

FIgURE 15: The influence of noise disturbances on robustness. 


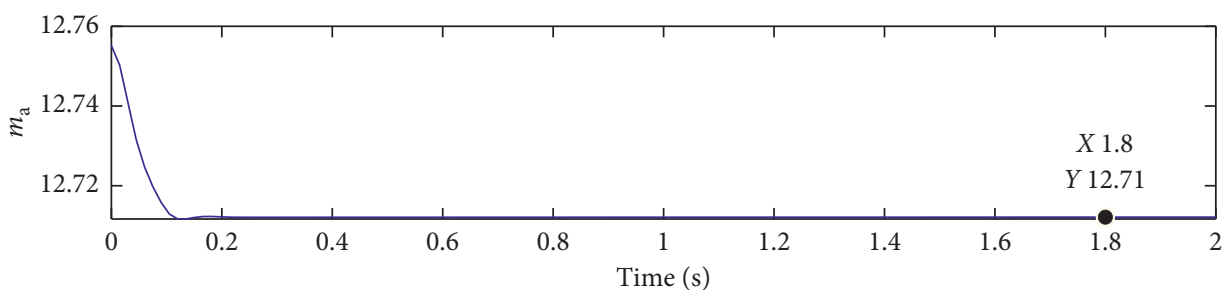

(a)

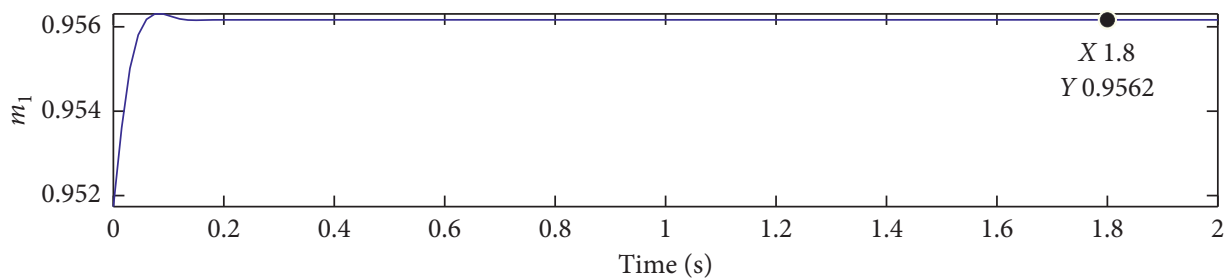

(b)

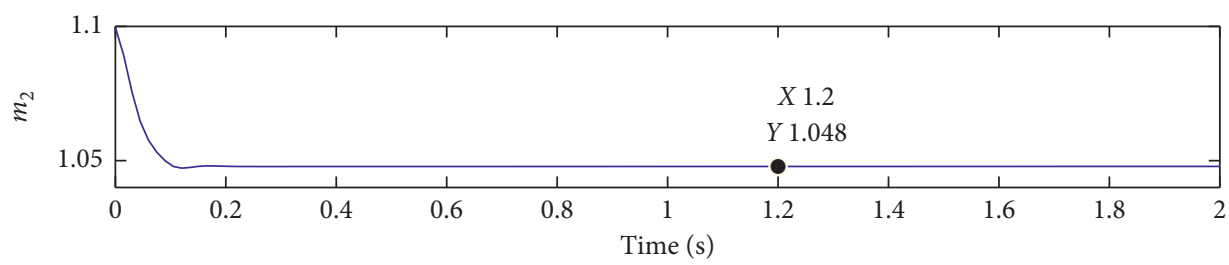

(c)

Figure 16: The estimated value of mass.

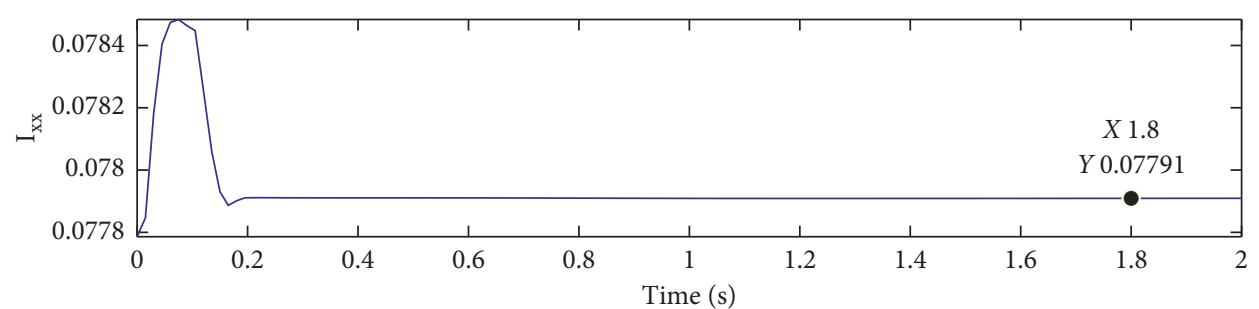

(a)

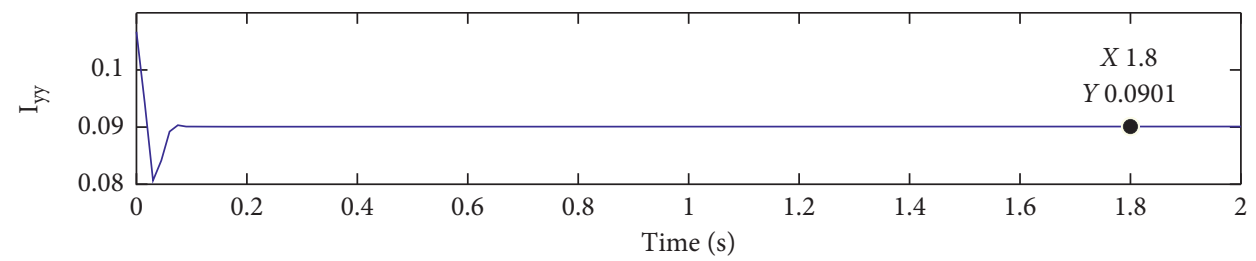

(b)

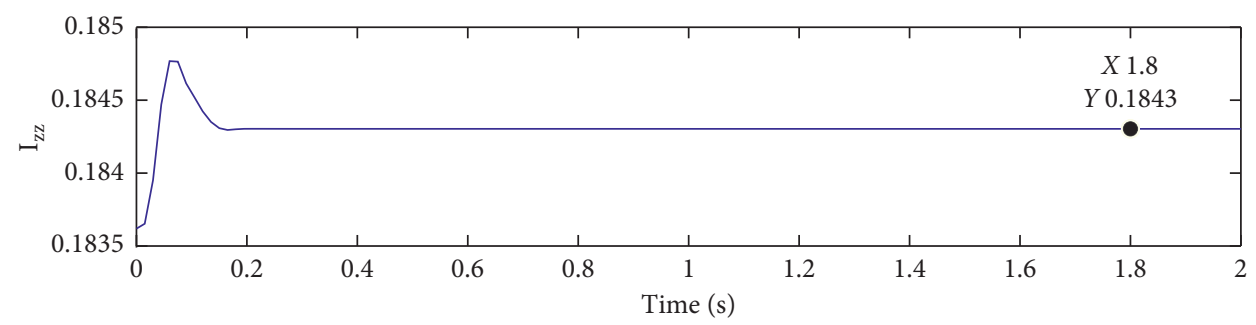

(c)

Figure 17: The estimated value of the moment of inertia of moving platform. 


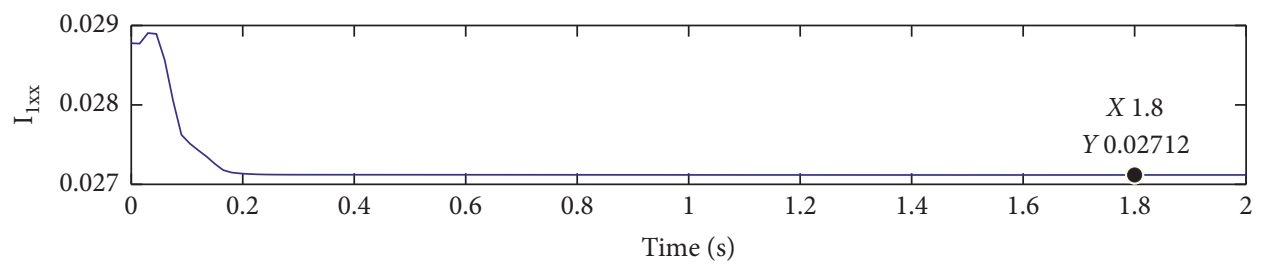

(a)

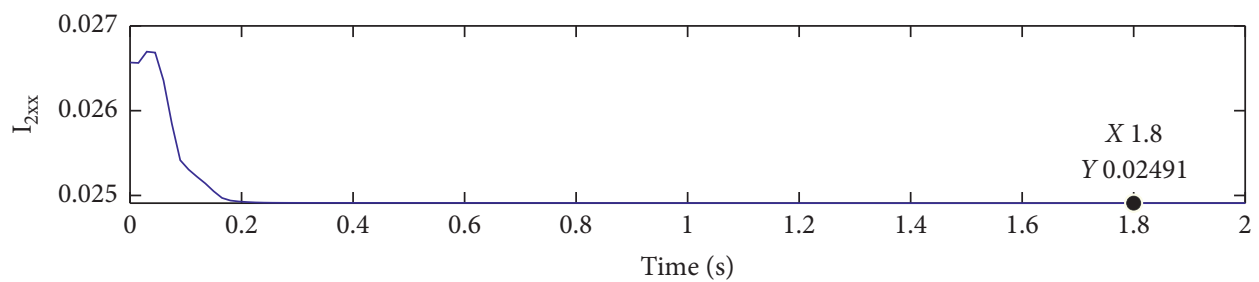

(b)

FIgURE 18: The moment of inertia of $\mathbf{I}_{x x, 1}$ and $\mathbf{I}_{x x, 2}$.

deviation before it reaches zero asymptotically. However, it is noticeable that the AS-SMC controller has a large variation nearby zero regions in the steady state compared with the AS-High Frequency controller, which illustrates that the ASSMC controller has a much wider range from zero regions than the AS-High Frequency controller, and these results are actually in accordance with the theoretical analysis. Three controllers have no particular rules in response time, but they are generally compatible systematically. As a whole, the AS-SMC controller has much greater errors, while the ASHigh Gain controller has much greater gain before the steady region, so the comparative analysis indicated that the AS-High Frequency controller has much better comprehensive performance.

In addition, the robustness simulation results depicted in Figure 15 demonstrate the reference trajectory tracking in $z$ position direction under the different noise cases, i.e., noise $=5$, noise $=10$, and noise $=40$. The local magnification illustrates that the high-frequency control system has approximate robust performance when the noise $=5$ and the noise $=10$, while the robust performance will be poor when the noise $=40$, which is mainly because the parameter $\rho=10$, and if the value of noise disturbances is greater than 10 , then the robustness will decrease.

From theoretical analysis, we know that adaptive control law has the advantage that the unknown parameter values can be estimated and compensated for by real-time estimation, and the robust terms of the controller can guarantee the system to maintain a considerable state with the presence of unknown external disturbances. Herein, we utilized the AS-High Frequency control to estimate the unknown parameters online. The partial results are shown in Figures 16-18. In Figure 16, the estimated parameter (i.e., $m_{\mathrm{a}} m_{1} m_{2}$ ) converges to actual value $[12.71,0.9562,1.048]^{T}$, and the nominal value in Table 1 is $[12.75,0.95,1.1]^{T}$; then the two results are approximately equivalent. In Figure 17, the unknown parameter is the moment of inertia of the moving platform, the estimated values converge to the actual parameter values [0.07791, 0.0901, $0.1843]^{T}$, and the nominal values are $[0.077,0.107,0.183]^{T}$. In
Figure 18, the estimated parameter (i.e., $\mathbf{I}_{1 x x}$ and $\mathbf{I}_{2 x x}$ ) is the moment of inertia in the $x$ direction of the upper limb and lower limb, and steady-state values of the unknown parameters converge to the estimation value $[0.02712,0.02491]^{T}$, while the nominal value is $[0.0288,0.0265]^{T}$. All the abovementioned estimated results illustrate that the actual values deviate from the nominal values within a small range due to ignoring manufacture tolerances, wear, friction, and so on. However, the proposed AS-High Frequency controller still can achieve satisfactory parameter estimation accuracy even without an accurate dynamic mathematic model, which greatly simplified the calculation process.

\section{Conclusions}

In this paper, we proposed three types of adaptive robust synchronous controllers for $2 \mathrm{RPU}-2 \mathrm{SPR}$ parallel manipulator with redundant actuation, the controllers are effective and stable when controlling the position and orientations of the platform, and the main contributions are following:

(1) The inverse kinematic analysis of the parallel manipulator has been formulated firstly, the dynamics formulation is derived in terms of the principle of virtual work, and then the dynamics expression can be further linear parameterization with regression matrix form.

(2) The three types of adaptive robust synchronous controllers are designed by incorporating the camera sensor technique into adaptive robust synchronous control architecture. Theoretical analysis implies the tracking errors, synchronization errors, and crosscoupling errors can converge to zero as were proved by utilizing the Lyapunov method.

(3) The simulation tests are conducted to demonstrate that the proposed three controllers can substantially improve the tracking performance when the controllers were implemented in real time. By comparative analysis, the adaptive robust synchronous high-frequency controller showed a better tracking 
performance among three controllers. In future works, to achieve higher accuracy for the parallel manipulator, the friction model should be further considered in the design of the controller, and the experiments will be executed on the prototype to verify the performance of the designed controller.

\section{Data Availability}

The data used to support the findings of this study are available from the corresponding author upon request.

\section{Conflicts of Interest}

The authors declare no conflicts of interest.

\section{Acknowledgments}

The authors would like to acknowledge the financial support of the Fundamental Research Funds for the Central Universities under grant nos. 2018JBZ007, 2018YJS136, and 2017YJS158, China Scholarship Council (CSC) under grant no. 201807090079. Meanwhile, Haiqiang Zhang is grateful to advanced robotics and mechatronics laboratory in York University and the science librarian John Dupuis.

\section{References}

[1] D. Zhang, Parallel Robotic Machine Tools, Springer, Berlin, Germany, 2010.

[2] M. D. Tafti, M. M. Moghaddam, and P. Torabi, "Kinematic analysis and mechatronic design of a 3-DOF parallel robot for craniotomy surgery," Modares Mechanical Engineering, vol. 17, no. 11, pp. 289-299, 2018.

[3] L. A. Castaneda, A. Luviano-Juarez, and I. Chairea, "Robust trajectory tracking of a Delta robot through adaptive active disturbance rejection control," IEEE Transactions on Control Systems Technology, vol. 23, no. 4, pp. 1387-1398, 2015.

[4] B. Hu, L. D. Zhang, and J. J. Yu, "Kinematics and dynamics analysis of a novel serial-parallel dynamic simulator," Journal of Mechanical Science and Technology, vol. 30, no. 11, pp. 5183-5195, 2016.

[5] C. Gosselin and L.-T. Schreiber, "Kinematically redundant spatial parallel mechanisms for singularity avoidance and large orientational workspace," IEEE Transactions on Robotics, vol. 32, no. 2, pp. 286-300, 2016.

[6] H. S. Kim, "Kinematically redundant parallel haptic device with large workspace," International Journal of Advanced Robotics Systems, vol. 9, no. 6, p. 260, 2012.

[7] L. J. Puglisi, Advanced control strategies for a 6 DOF hydraulic parallel robot based on the dynamic model, Ph.D. thesis, Universidad Politecnica de Madrid, Madrid, Spain, 2016.

[8] Y. Li and Q. Xu, "Dynamic modeling and robust control of a 3-PRC translational parallel kinematic machine," Robotics and Computer-Integrated Manufacturing, vol. 25, no. 3, pp. 630-640, 2009.

[9] X. Liu, Y. Xu, J. Yao, J. Xu, S. Wen, and Y. Zhao, "Controlfaced dynamics with deformation compatibility for a 5-DOF active over-constrained spatial parallel manipulator 6PUSUPU," Mechatronics, vol. 30, pp. 107-115, 2015.

[10] S. H. Wen, J. H. Chen, G. Q. Qin, Q. Zhu, and H. Wang, “An improved fuzzy model predictive control algorithm based on the force/position control structure of the five-degree of freedom redundant actuation parallel robot," International Journal of Advanced Robotic Systems, vol. 15, no. 5, 2018.

[11] M. Sharifzadeh, M. Tale Masouleh, A. Kalhor, and P. Shahverdi, "An experimental dynamic identification \& control of an overconstrained 3-DOF parallel mechanism in presence of variable friction and feedback delay," Robotics and Autonomous Systems, vol. 102, pp. 27-43, 2018.

[12] A. Muller, "Redundant actuation of parallel manipulators," in Parallel manipulators, Towards New Applications, vol. 4London UK, IntechOpen, 2008.

[13] H. Liu, T. Huang, A. Kecskeméthy, D. G. Chetwynd, and Q. Li, "Force/motion transmissibility analyses of redundantly actuated and overconstrained parallel manipulators," Mechanism and Machine Theory, vol. 109, pp. 126-138, 2017.

[14] J. Yao, W. Gu, Z. Feng, L. Chen, Y. Xu, and Y. Zhao, "Dynamic analysis and driving force optimization of a 5-DOF parallel manipulator with redundant actuation," Robotics and Computer-Integrated Manufacturing, vol. 48, pp. 51-58, 2017.

[15] R. Boudreau, J. Léger, H. Tinaou, and A. Gallant, "Dynamic analysis and optimization of a kinematically redundant planar parallel manipulator," Transactions of the Canadian Society for Mechanical Engineering, vol. 42, no. 1, pp. 20-29, 2018.

[16] R. Boudreau and S. Nokleby, "Force optimization of kinematically-redundant planar parallel manipulators following a desired trajectory," Mechanism and Machine Theory, vol. 56, pp. 138-155, 2012.

[17] X. Wang, S. Guo, H. B. Qu, and F. Q. Zhao, "Optimal allocation method of parallel mechanism and its application," Journal of Mechanical Engineering, vol. 55, no. 1, pp. 32-41, 2019.

[18] L. Ren, J. K. Mills, and D. Sun, "Adaptive synchronized control for a planar parallel manipulator: theory and experiments," Journal of Dynamic Systems, Measurement, and Control, vol. 128, no. 4, pp. 976-979, 2006.

[19] W. Shang and S. Cong, "Nonlinear adaptive task space control for a 2-DOF redundantly actuated parallel manipulator," Nonlinear Dynamics, vol. 59, no. 1-2, pp. 61-72, 2010.

[20] R. Babaghasabha, M. A. Khosravi, and H. D. Taghirad, "Adaptive control of KNTU planar cable-driven parallel robot with uncertainties in dynamic and kinematic parameters," in Proceedings of the Second International Conference on CableDriven Parallel Robots, vol. 32, pp. 145-159, Cham, Switzerland, November 2015.

[21] D. Zhang and B. Wei, "A review on model reference adaptive control of robotic manipulators," Annual Reviews in Control, vol. 43, pp. 188-198, 2017.

[22] L. Wang, Z. Lu, X. P. Liu, K. Liu, and D. Zhang, "Adaptive control of a parallel robot via backstepping technique," International Journal of Systems Control and Communications, vol. 1, no. 3, pp. 312-324, 2005.

[23] M. Honegger, A. Codourey, and E. Burdet, "Adaptive control of the hexaglide, a 6 DOF parallel manipulator," in Proceedings of the 1997 IEEE international Conference on Robotics and Automation, Albuquerque, NM, USA, April 1997.

[24] D. Y. Zhao, S. Y. Li, and F. Gao, "Fully adaptive feedforward feedback synchronized tracking control for Stewart platform systems," International Journal of Control, Automation, and System, vol. 6, no. 5, pp. 689-701, 2008.

[25] J. Shi, J. Yao, T. M. Li, and Y. S. Du, "Learning-based optimal desired compensation adaptive robust control for a flexure-based micromotion manipulator," Applied Sciences, vol. 7, no. 4, p. 406, 2017.

[26] E. Yime, R. Saltaren, and J. Diaz, "Robust adaptive control of the Stewart-Gough robot in the task space," in Proceedings of the 2010 American Control Conference, pp. 5248-5253, Marriott Waterfront, Baltimore, MD, USA, June 2010. 
[27] X. C. Zhu, G. L. Tao, B. Yao, and J. Cao, "Adaptive robust posture control of parallel manipulator driven by pneumatic muscles with redundancy," IEEE/ASME Transactions on Mechatronics, vol. 13, no. 4, pp. 441-450, 2008.

[28] S. Roy, S. B. Roy, and I. N. Kar, "Adaptive-robust control of Euler-Lagrange systems with linearly parametrizable uncertainty bound," IEEE Transactions on Control Systems Technology, vol. 26, no. 5, pp. 1842-1850, 2018.

[29] M. Bennehar, Some contributions to nonlinear adaptive control of PKMs: from design to real-time experiments, Ph.D. thesis, Université de Montpellier, Montpellier, France, 2015.

[30] W. W. Shang and S. Cong, "Dexterity and adaptive control of planar parallel manipulators with and without redundant actuation," Journal of Computational and Nonlinear Dynamics, vol. 10, no. 1, Article ID 011002, 2015.

[31] A. T. Vo and H. J. Kang, "An adaptive neural non-singular fast-terminal sliding-mode control for industrial robotic manipulators," Applied Sciences, vol. 8, no. 12, p. 2562, 2018.

[32] H. Zhang, H. Fang, D. Zhang, X. Luo, and Q. Zou, "Forward kinematics and workspace determination of a novel redundantly actuated parallel manipulator," International Journal of Aerospace Engineering, vol. 2019, Article ID 4769174, 14 pages, 2019.

[33] G. Shen, Y. Tang, J. Zhao, H. Lu, X. Li, and G. Li, "Jacobian free monotonic descent algorithm for forward kinematics of spatial parallel manipulator," Advances in Mechanical Engineering, vol. 8, no. 4, 2016.

[34] H.-Q. Zhang, H.-R. Fang, B.-S. Jiang, and S.-G. Wang, "Dynamic performance evaluation of a redundantly actuated and over-constrained parallel manipulator," International Journal of Automation and Computing, vol. 16, no. 3, pp. 274-285, 2019.

[35] S. Roy and S. Baldi, "On reduced-complexity robust adaptive control of switched Euler-Lagrange systems," Nonlinear Analysis: Hybrid Systems, vol. 34, pp. 226-237, 2019.

[36] S. Roy and S. Baldi, "A simultaneous adaptation law for a class of nonlinearly parametrized switched systems," IEEE Control Systems Letters, vol. 3, no. 3, pp. 487-492, 2019.

[37] R. Ortega and M. W. Spong, "Adaptive motion control of rigid robots: a tutorial," in Proceedings of the 27th IEEE Conference on Decision and Control, pp. 1575-1584, Austin, TX, USA, December 1988.

[38] T.-S. Zhan and C.-C. Kao, "Modified PSO method for robust control of 3RPS parallel manipulators," Mathematical Problems in Engineering, vol. 2010, Article ID 302430, 25 pages, 2010.

[39] C. Yang, Q. Huang, H. Jiang, O. Ogbobe Peter, and J. Han, "PD control with gravity compensation for hydraulic 6-DOF parallel manipulator," Mechanism and Machine Theory, vol. 45, no. 4, pp. 666-677, 2010.

[40] D. Shiferaw, A. Jain, and R. Mitra, "High performance control of Stewart platform manipulator using sliding mode control with synchronization error," International Journal of Intelligent Mechatronics and Robotics, vol. 1, no. 4, pp. 19-43, 2011.

[41] P. K. Jamwal, S. Q. Xie, Y. H. Tsoi, and K. C. Aw, "Forward kinematics modelling of a parallel ankle rehabilitation robot using modified fuzzy inference," Mechanism and Machine Theory, vol. 45, no. 11, pp. 1537-1554, 2010.

[42] F. Piltan, A. Taghizadegan, and N. B. Sulaiman, "Modeling and control of four degrees of freedom surgical robot manipulator using MATLAB/SIMULINK," International Journal of Hybrid Information Technology, vol. 8, no. 11, pp. 47-78, 2015.
[43] S. Yao, H. Li, L. Zeng, and X. Zhang, "Vision-based adaptive control of a 3-RRR parallel positioning system," Science China Technological Sciences, vol. 61, no. 8, pp. 1253-1264, 2018.

[44] A. Traslosheros, J. Sebastián, J. Torrijos, R. Carelli, and E. Castillo, "An inexpensive method for kinematic calibration of a parallel robot by using one hand-held camera as main sensor," Sensors, vol. 13, no. 8, pp. 9941-9965, 2013.

[45] G. L. Wu, S. P. Bai, J. A. Kepler, and S. Caro, "Error modelling and experimental validation of a planar 3-PPR parallel manipulator with joint clearances," Journal of Mechanisms and Robotics, vol. 4, no. 4, Article ID 041008, 2012.

[46] https://www.mathworks.com/help/vision/ug/stereo-cameracalibrator-app.html.

[47] http://emanual.robotis.com/.

[48] A. Escalera and J. M. Armingol, "Automatic chessboard detection for intrinsic and extrinsic camera parameter calibration," Sensors, vol. 10, no. 3, pp. 2027-2044, 2010. 